
. 9

\title{
A multi-method, multi-scale theoretical study of He and Ne
}

\section{diffusion in zircon}

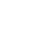

Cécile Gautheron ${ }^{1 *}$, Duval Mbongo Djimbi ${ }^{2}$, Jérôme Roques ${ }^{2}$, Hilal Balout ${ }^{1,2}$, Richard A. Ketcham ${ }^{3}$, Eric Simoni², Raphael Pik ${ }^{4}$, Anne-Magali Seydoux-Guillaume ${ }^{5,6}$, (1) Laurent Tassan-Got ${ }^{2}$

1- GEOPS, CNRS, Univ. Paris-Sud, Université Paris-Saclay, Rue du Belvédère, Bât. 504,

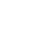 F-91405 Orsay, France

2- Institut de Physique Nucléaire d'Orsay, CNRS-IN2P3, Univ. Paris-Sud, Université ParisSaclay, 91406 Orsay cedex, France

3- Jackson School of Geosciences, The University of Texas at Austin, Austin, Texas, U.S.A. 4- CRPG, 15 rue ND des pauvres BP20, 54501 Vandoeuvre-Lès-Nancy Cedex, France 5- Université Clermont Auvergne, CNRS, IRD, OPGC, Laboratoire Magmas et Volcans, F63000 Clermont-Ferrand, France 6- Univ Lyon, UJM-Saint-Etienne, UCA, CNRS, IRD, LMV UMR 6524, F-42023 SaintEtienne, France 18 * corresponding author, cecile.gautheron@u-psud.fr

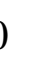




\section{Abstract}

The quantification of $\mathrm{He}$ and $\mathrm{Ne}$ diffusion behavior in crystals rich in $\mathrm{U}$ and $\mathrm{Th}$ such as zircon is key for the interpretation of $(\mathrm{U}-\mathrm{Th}) /{ }^{4} \mathrm{He}$ and $(\mathrm{U}-\mathrm{Th}) /{ }^{21} \mathrm{Ne}$ thermochronometric ages. Multiple parameters such as chemical substitution, channel obstruction and damage can modify the diffusivity compared to a pristine structure. To investigate the impact of these parameters, we have conducted a theoretical diffusion study combining a series of methods and approaches to address the problem across the necessary range of scales (atomic to crystal size). First, using quantum calculation, we determine the different $\mathrm{He}$ and $\mathrm{Ne}$ insertion sites, insertion energies and diffusion pathways at the atomic scale for an ideal pristine zircon structure (i.e. damage free). These results serve as input for a 3D random walk simulation of atomic trajectories that provides diffusion coefficients for damage-free zircon crystals. Second, as natural zircon crystals are not perfect, we model the impact of different types of damage and diffusion pathway obstruction at the atomic level on $\mathrm{He}$ and $\mathrm{Ne}$ diffusion in 3D. The calculated $\mathrm{He}$ and $\mathrm{Ne}$ diffusion coefficients for pure $\mathrm{ZrSiO}_{4}$ exhibit strongly anisotropic behavior and very high diffusivity along the $c$-axis, and with $3 \mathrm{D}$, closure temperatures of $-197^{\circ} \mathrm{C}$ and $-202{ }^{\circ} \mathrm{C}$ respectively. The results for He are comparable to previous DFT studies but strongly different from experimental diffusion results; results for $\mathrm{Ne}$ are similar in this respect. Modelling the impact of different types of damage (vacancies, recoil, fission, voids or fluid inclusions) and obstruction on $\mathrm{He}$ and Ne diffusion reveals important implications for the (U-Th)/He and (U$\mathrm{Th} / \mathrm{Ne}$ thermochronometers. First, obstruction alone does not significantly modify $\mathrm{He}$ and $\mathrm{Ne}$ diffusion except to reduce anisotropy. Second, trapping is the primary mechanism altering He and $\mathrm{Ne}$ diffusion even at low dose, and we predict the maximal trapping energies for He and $\mathrm{Ne}$ to be 164 and $320 \mathrm{~kJ} / \mathrm{mol}$, similar to values inferred from experimental data. We also propose that the closure temperature increases non-linearly with damage, with effective trapping energy increasing with dose until a threshold, possibly corresponding to a percolation 
transition, after which retentivity decreases. Based on field data sets we also anticipate a value for this threshold of around $\sim 2-5 \times 10^{17} \alpha / g$, lower than previously proposed. We show Ne to be highly blocked by damage and predict similar diffusion behavior to $\mathrm{He}$, but with higher retentivity. We demonstrate the importance of investigating rare gas diffusion at the atomic level for comparison with experimental data, in order to build a predictive diffusion law at different scales.

\section{Introduction}

In the last two decades, radiogenic ${ }^{4} \mathrm{He}$ and nucleogenic ${ }^{21} \mathrm{Ne}$ have been used for (U$\mathrm{Th}) /{ }^{4} \mathrm{He}$ and $(\mathrm{U}-\mathrm{Th}) /{ }^{21} \mathrm{Ne}$ thermochronology in different minerals such as apatite, zircon and iron oxides for a broad range of geological applications (e.g. Farley and Flowers, 2012; Farley and Stockli, 2002; Gautheron et al., 2006; Reiners et al., 2002; Shuster et al., 2005; Zeitler et al., 1987). Robust He and Ne age interpretation strongly depends on quantitative knowledge of diffusion behavior in crystals, which is linked to the closure temperature Tc (Dodson, 1973), representing to first order the temperature below which $\mathrm{He}$ and $\mathrm{Ne}$ are significantly retained during cooling. He and Ne diffusion in crystals theoretically follows a simple Arrhenius law with isotropic or anisotropic diffusion. However, it has been shown that, for apatite and zircon, diffusion behavior is highly variable. Experimental data sets have Tc values ranging from 40 to $120^{\circ} \mathrm{C}$ for $\mathrm{He}$ in apatite (Shuster et al., 2006) and from -60 to $190^{\circ} \mathrm{C}$ for zircon (Guenthner et al., 2013, and references therein). For Ne diffusion in zircon, data are sparse due to analytical difficulties, and Tc estimates come only from indirect assessment and one published abstract (Gautheron et al., 2006; van Soest et al., 2013).

Radiation damage is considered to be a primary contributor to this variability, although the impacts of damage type (alpha, recoil and fission damage) and topology (Frenkel-pair, large damage and amorphous zone) on He diffusion in zircon are not well defined. In addition to forming traps for diffusing elements (Farley, 2000), damage can also change diffusive behavior 
for even low-damage zircon by reducing the strong anisotropy along the $c$-axis (Bengtson et al., 2012; Farley, 2007). Similar complications may arise from other imperfections such as inclusions, voids, and defects (Zeitler et al., 2017; Danišík et al., 2017).

The relationship between He diffusivity and dose in zircon suggests another topological effect. Diffusivity has been shown to decrease with increasing dose until a threshold is reached, after which it strongly increases with further damage (Guenthner et al., 2013), although recent work suggests that this threshold dose may vary (Anderson et al., 2017). This flip in behavior may be linked to the onset of percolation, in which overlapping damage zones start to form an interconnected network (Salje et al., 1999), essentially dividing the crystal into two transport regimes, one through normal crystal (albeit with imperfections) and one through the damage network (Ketcham et al., 2013). Superimposed on the complexity of diffusion is the process of annealing, which changes the landscape in which diffusion occurs, as it is happening. Recent work suggests that damage annealing in zircon also may depend on dose (Ginster et al., 2019), further highlighting the challenges to understanding the regime in which diffusion operates.

As a result of this complexity, there are limits to how much progress can be made using exclusively empirical observations and experiments on incompletely-understood natural materials (e.g., Reiners et al., 2004; Reiners, 2005; Cherniak et al., 2009; Guenthner et al., 2013; Anderson et al., 2017; Ginster et al., 2019; Zeitler et al., 2017; Danišík et al., 2017). A rigorous physical understanding of the diffusion process and how it is affected by imperfections is an indispensible complement for the varied and extensive data gathering currently underway. Although the inevitably chaotic and variable nature of damage zones makes them hard to characterize, transport of $\mathrm{He}$ and $\mathrm{Ne}$ in the areas between damage zones, and between damaged and undamaged crystal, is accessible through theoretical and computational approaches.

Several studies have been conducted using Density Functional Theory (DFT) calculations to quantify the He diffusion coefficient in damage-free zircon (Reich et al., 2007; 
Saadoune and De Leeuw, 2009; Saadoune et al., 2009). Independent studies consistently find that $\mathrm{He}$ is not expected to be retained in the zircon structure at Earth-surface temperatures; for example, Bengtson et al. (2012) calculate $\mathrm{T}_{\mathrm{c}} \sim-150^{\circ} \mathrm{C}$ along the crystallographic $c$-axis. However, recent DFT studies on He and Ne diffusion in apatite and hematite give results more similar to experimental data on real specimens, supporting the veracity and applicability of this theoretical approach (Balout et al., 2017a; Djimbi et al., 2015; Gerin et al., 2017).

In this contribution, we investigate $\mathrm{He}$ and $\mathrm{Ne}$ diffusion in zircon from atomic to grain scales using a coordinated series of methodologies developed in recent studies (Djimbi et al., 2015, Balout et al., 2017a, b; Gerin et al., 2017) to examine important factors that can modify diffusion rates, including trapping, anisotropy, and path obstruction. As no published $\mathrm{Ne}$ diffusion data exist, we use DFT at the atomic scale to build and optimize the ideal zircon unit cell accounting for quantum features, and we inventory the insertion and interstitial sites. Once these sites are identified, we combine the DFT approach with the Nudged Elastic Band (NEB) method to determine the migration energies between them. We complete our atomic-scale characterization using Transition State Theory (TST) to compute jump rates between sites. To extend to a larger scale spanning defects, DFT and TST outputs are used in a Kinetic Monte Carlo (KMC) simulation to determine the diffusion trajectories and the effective diffusion coefficients in 3D. We then compare our results with published experimental data and investigate the processes that can modify $\mathrm{He}$ and $\mathrm{Ne}$ diffusion in zircon. We simulate and evaluate different hypotheses, such as trapping of $\mathrm{He}$ and $\mathrm{Ne}$ in damage, and diffusion pathway obstruction, in 3D, and their impact on closure temperature. Finally, we discuss the model implications for the (U-Th)/He and (U-Th)/Ne thermochronometers.

\section{Methods, the multi-scale approach}

We first provide a general summary of our methods and how they interact with each other, as illustrated in Figure 1. Density Functional Theory (DFT) is a computational modeling 
method to calculate the energy of interacting atoms by calculating their external electronic

125 configuration. This is done by solving the Schrödinger equation for the electrons using

126 functionals of the electron density. The DFT just solves static problems, atoms at given

127 positions, and does not include dynamics, contrary to molecular dynamics. However, in contrast

128 to molecular dynamics it includes the quantum effects for the electrons, thereby providing the

129 most accurate energy estimation of an ensemble of interacting atoms. Due to the high

130 calculation time, requiring cluster computing, the number of considered atoms remains small

131 (<150 atoms) compared to a real crystal, and therefore a super-cell containing a small number

132 of motionless atoms is used and an infinite repetition of this super-cell is assumed to represent

133 an infinite lattice. The stable position of the atoms for a crystal lattice is found by nudging atom

134 positions until the total energy is minimized. In the same way, an insertion site for $\mathrm{He}$ or $\mathrm{Ne}$ is

135 found by inserting it into a pre-computed lattice and finding the positions with the minimum energy with respect to displacement after relaxation of the neighboring atoms.

$\mathrm{He}$ and $\mathrm{Ne}$ atoms can statistically jump between insertion sites when they vibrate as a consequence of the finite temperature. This is a dynamical process, beyond the scope of DFT,

139 which addresses static configurations only. Therefore, another approach has to be followed,

140 namely the Transition State Theory (TST), which computes the jumps statistically. It is based on a full mapping of the energy field encountered when the inserted atom moves along its path to its neighboring site, as delivered by the DFT analysis. This approach is valid because the

143 Born-Oppenheimer approximation allows consideration of the motion of atoms as quasi-

144 stationary from the electron viewpoint.

145 The ideal pristine crystal represented by DFT and TST does not capture all the 146 properties of a natural one, which will always contain defects, distortions, dislocations and 147 substitutions, and typically mineral and/or fluid inclusions. However, even in a highly degraded 148 crystal, diffusion occurs in pristine lattice for at least some length scale. The approach we 
149 develop here is to consider the ideal case and see how it should be amended when alterations

150 of the pristine lattice are added. Only when significant amorphisation takes place does this 151 approach become invalid. The alteration of the ideal lattice by vacancies, interstitial atoms, and

152 substitutions breaks the periodicity of the lattice and introduces heterogeneity over length scales 153 much larger than the lattice cell dimension. Their effects on diffusion depend on their energy 154 and their topology. Therefore, the easiest way to study them is the simulation of diffusion by 155 random walk over many lattice cells spanning many defects. For this we use the Kinetic Monte 156 Carlo method (KMC), which operates at the mesoscopic level and delivers diffusion coefficients for a heterogeneous medium, based on the energetics of the DFT and the transition rates of the TST (Figure 1). Finally, to compute $\mathrm{He}$ and $\mathrm{Ne}$ ages in crystals the diffusion

159 equation has to be solved at the macroscopic level using the diffusivities delivered by KMC, or 160 directly by DFT and TST in case of an ideal crystal. In summary, DFT calculates the mapping of energy as a function of atom location. TST

162 then computes the transition rates by using this mapping and by making use of thermodynamic 163 equilibrium in phase space. KMC then quantifies the effect of randomly distributed alterations 164 of the crystal-like traps or obstructions, independently of the mechanism producing them 165 (interstitial atoms, substitutions, etc.). This pipeline delivers bulk diffusion coefficients for 166 solving the 3D diffusion equation for a finite grain, as illustrated in Figure 1.

\subsection{Density Functional Theory}

Following the approach described in detail by Djimbi et al. (2015), we use the Periodic

169 Density Functional Theory (DFT; Hohenberg and Kohn, 1964; Kohn and Sham, 1965), as 170 implemented in the Vienna Ab-initio Simulation Package (VASP) (Kresse and Furthmüller, 171 1996; Kresse and Hafner, 1993), for all structural optimization and insertion site investigations 172 of both He- and Ne-doped zircon lattices. All parameters were optimized on the zircon unit cell, 173 and the relaxed unit cell geometrical parameters are in good agreement with experimental 
174 values. To minimize the volume relaxation effect due to the incorporation of $\mathrm{Ne}$ and $\mathrm{He}$ atoms,

175 and to avoid interactions between $\mathrm{He}$ or $\mathrm{Ne}$ with neighboring cells, the zircon unit cell was 176 duplicated along each spatial direction, resulting in a $2 \times 2 \times 2$ super cell consisting of 192 atoms

177 (Figure 2A and 2B). We determine the insertion energy of $\mathrm{He}$ and $\mathrm{Ne}$ in each interstitial site by 178 relaxing the structure with the help of the gradient technique at constant volume. The insertion 179 energy is defined as the energy needed to bring the $\mathrm{He}$ or $\mathrm{Ne}$ from outside the crystal into the 180 interstitial site. It is calculated as:

$$
E_{\text {ins }}=E_{\text {He+zircon }}-\left(E_{\text {zircon }}+E_{H e}\right)
$$

182 where $E_{H e+z i r c o n}, E_{\text {zircon }}$ and $E_{H e}$ are respectively the absolute energy of the system with $\mathrm{He}$ 183 or $\mathrm{Ne}$ in the zircon host lattice, the absolute energy of the host lattice, and the energy of the 184 isolated $\mathrm{He}$ or $\mathrm{Ne}$ atom. We use the Nudged Elastic Band (NEB) (Jónsson et al., 1998; Mills et al., 1995) method to determine the migration energy between insertion sites. NEB is an efficient approach to estimating the minimum energy path (MEP) and the migration energy between two interstitial

sites $\left(E_{m i g}^{i j}\right)$, which is defined as the difference in energy from when the atom is at the saddle point to when it is in the insertion site. It is also called the barrier energy. The NEB is a chainof-states method (Elber and Karplus, 1987; Pratt, 1986) found by constructing a series of system images between states. Adjacent images are linked by a spring force to ensure the continuity of the path, thus mimicking an elastic band. In this study, all NEB calculations are performed with a spring constant of $-5 \mathrm{eV} / \AA^{2}$.

\subsection{Transition State Theory} Interstitial diffusion occurs through a random walk of an atom from one site in the crystal lattice to another neighboring free one. The atom needs enough energy to accomplish this motion; as there is an energy barrier to overcome, diffusion is a thermally activated process. 
198 As already mentioned, this dynamical process cannot be directly calculated by DFT, which

199 addresses static configurations. However, as the motion occurs in thermodynamic equilibrium 200 the canonical ensemble is assumed in phase space. This leads to the transition state theory 201 (Vineyard, 1957; Voter, 1986; Wert and Zener, 1949), in which the atomic motion is controlled 202 by the jump probabilities, $\Gamma$, which depend exponentially on temperature.

$$
\Gamma^{i j}=v_{0} e^{-\frac{E_{m i g}^{i j}}{k_{B} T}}
$$

$$
\text { With } v_{0}=\frac{\prod_{i=1}^{3 N} v_{i}^{\text {init }}}{\prod_{i=1}^{3 N-1} v_{i}^{*}} \stackrel{\text { Vineyard approx. }}{=} \approx \frac{\prod_{i=1}^{3} v_{i}^{\text {init }}}{\prod_{i=1}^{2} v_{i}^{*}}
$$

$$
\text { And } E_{m i g}^{i j}=E^{*}-E^{i n i t}
$$

Where $v_{0}$ is the attempt frequency; $v_{i}^{\text {init }}$ and $v_{i}^{*}$ are the normal mode frequencies at,

respectively, the initial and transition states; $E_{m i g}^{i j}$ represents the migration energies; and $N$ is 208 the number of atoms in the system. For a molecule with $N$ atoms there are $3 N$ degrees of 209 freedom. For a non-linear molecule three degrees of freedom can be assigned to translation of 210 a body as a whole $\left(T_{x}, T_{y}, T_{z}\right)$ and three to rotations $\left(R_{x}, R_{y}, R_{z}\right)$. The remaining motions of the 211 atoms are displacements of the atoms from their mean positions. These fundamental vibrations 212 are referred to as "normal modes". Thus, a non-linear molecule has $3 N-6$ normal modes. In the 213 insertion location, the energy is minimum, and therefore the energy has an upward curvature 214 for any displacement of the inserted atom. This 3D-curvature can be expressed with three 215 eigenvalues, which translate directly to the three normal frequencies. This translation is exactly 216 the same as for any harmonic oscillator like a pendulum. At the saddle point, the same features 217 apply, but only transverse motion relative to the transition path is involved, and therefore there 218 are two frequencies. According to the Vineyard (1957) approximation, for one atom (He or $\mathrm{Ne})$, 219 the energetics of other atoms are not affected by the position of the migrating atom being 220 considered. The TST allows thus provides a well-understood theoretical approach for 221 calculating the frequency factor (Figure 1). 


\subsection{Kinetic Monte Carlo Method}

In a pristine lattice, the activation energy $\left(\mathrm{E}_{\mathrm{a}}\right)$ corresponds to the migration energy obtained from DFT, and the frequency factor $\left(\mathrm{D}_{0}\right)$ can be extracted directly from the TST formulas (Fig. 1). However, when obstructions occur the diffusivity depends on their topology, and a simulation over macroscopic crystals has to be run to determine the modified diffusion coefficients. The Kinetic Monte Carlo (KMC) method (Bortz et al., 1975; Gillespie, 1976) is particularly apt for our purposes because it can be used to extend simulation to time scales far beyond the vibrational period and lengths much larger than the cell size. The KMC simulates, at a given temperature, a random walk over the interstitial sites based on the microscopic jump probabilities $(\Gamma)$, which have been calculated from TST using the DFT results (Fig. 1). In addition, it takes into account potential obstructions and traps, as well as anisotropy.

For a selected temperature, one $\mathrm{He}$ (or $\mathrm{Ne}$ ) atom is placed randomly in one available insertion site. For the possible diffusion directions, with the calculated jumping frequencies given by the TST, we randomly choose a number to simulate the jumping time for each direction. The distribution of jumping times follows an exponential decay. The jump direction with the lowest time is chosen, the time and coordinate are updated, and the time is added to the total travel time. This is repeated $m$ times for each atom, and $n$ trajectories (atoms) are simulated, using the method of Djimbi et al. (2015). For each trajectory, the total time is computed as the sum of the residence times in each site between jumps. The diffusion coefficient is obtained by averaging over the ensemble of $n$ trajectories. If $\left\langle x^{2}\right\rangle,\left\langle y^{2}\right\rangle,\left\langle z^{2}\right\rangle$ are the averages over the trajectories of the squares of distances between initial and final points in $x, y$ and $z,\langle t\rangle$ is the average time of the trajectories, and if we denote as $D_{x}, D_{y}, D_{z}$ the diffusion coefficient along the axes, then according to the Einstein relation.

$$
D_{x}=\frac{<x^{2}>}{2<t>}
$$




$$
D_{y}=\frac{<y^{2}>}{2<t>}
$$

$$
D_{z}=\frac{<z^{2}>}{2<t>}
$$

From the above relations the diffusion coefficients can be assessed even in the case of anisotropic diffusion, as is necessary for zircon. The temperature dependence is obtained by running the random walk at different temperatures.

To account for highly anisotropic behavior along the $c$ axis and its possible obstruction,

252 the random walk operates on a sub-lattice of $128 \times 128 \times 4096$ interstitial sites. When an atom 253 jumps against an edge of the sub-lattice it is automatically re-inserted into the opposite side, so that the geometry can be considered as infinite with a periodicity given by the size of the sublattice. Obstructions are simulated by inhibiting some fraction of sites randomly distributed over the sub-lattice.

\section{$257 \quad 2.4 \quad 3 D$ diffusion modeling}

Finally, these two descriptions provide a bulk diffusion coefficient, which is used to calculate the diffusion over a finite grain where the surface is taken into account by solving in 3D the diffusion equation appropriate to surface boundary conditions. A modified version of

261 the rare gas 3D diffusion code published in Gautheron and Tassan-Got (2010) and used in

262 subsequent studies (Djimbi et al., 2015; Gautheron et al., 2009; 2012) has been implemented to

263 simulate $\mathrm{He}$ and $\mathrm{Ne}$ diffusion in zircon. This code solves the diffusion equation by a Monte 264 Carlo method at the macroscopic level, using the diffusivity extracted from the KMC 
simulation. This approach accounts for crystal surface boundaries, which is not possible in the

266 KMC code.

\section{Results}

\subsection{Investigation of $\mathrm{He}$ and Ne interstitial sites}

Zircon crystallizes as a body-centered tetragonal unit cell, with a structure consisting of a chain of alternating edge-sharing $\left(\mathrm{SiO}_{4}\right)$ tetrahedral and $\left(\mathrm{ZrO}_{8}\right)$ triangular dodecahedra parallel to $c$-axis (Figure 2A and 2B). The lateral connection of these chains provides two types of possible sites $\left(\mathrm{S}_{1}\right.$ and $\left.\mathrm{S}_{1}{ }^{\prime}\right)$ into which $\mathrm{He}$ or $\mathrm{Ne}$ atoms can be inserted; these insertion site positions, and the diffusion pathways between them, are identical $\mathrm{He}$ and $\mathrm{Ne}$. The connection of these interstitial sites provides open channels along each direction in which He or Ne could diffuse (Figure 2A' and 2B'). In this part of our analysis, a periodic-DFT is run first to build an optimized model of zircon. Next, the model is used to identify diffusion channels and all possible interstitial insertion sites for both $\mathrm{He}$ and $\mathrm{Ne}$ atoms. As mentioned above, a $2 \times 2 \times 2$ supercell was optimized as the host lattice (Figure 2), which was large enough to simulate and study the $\mathrm{He}$ and $\mathrm{Ne}$ insertion in the zircon crystal lattice free of boundary effects.

The $c$-axis channel is formed by alternating edge-sharing $\mathrm{SiO}_{4}$ tetrahedron and $\mathrm{ZrO}_{8}$ dodecahedron units (Figure 2A), and Figure 2A' illustrates the different $\mathrm{He}$ (or $\mathrm{Ne}$ ) insertion sites along this pathway. In each $c$-channel four sites are inventoried for each lattice cell and they are energetically identical due to the symmetries of the crystal. They are spaced by $1.5 \AA$. Two types of sites are recognized: $\mathrm{S}_{1}$, which allows a jump to an adjacent $c$ channel along $a$ (jump along $b$ forbidden), and $\mathrm{S}_{1}$ ', which allows a jump along $b$ to an adjacent $c$ channel. Along $c$, the sites form a sequence $\mathrm{S}_{1}-\mathrm{S}_{1}{ }^{\prime}-\mathrm{S}_{1}-\mathrm{S}_{1}{ }^{\prime} \ldots$ (Figure $2 \mathrm{~A}^{\prime}$ ). Therefore, along $a$, diffusion pathways connect $\mathrm{S}_{1}$ sites. The same applies along $b$ with the $\mathrm{S}_{1}$ ' sites. The channels along the $a$ - and $b$-axis are energetically identical and they will be noted $a / b$-(axis or channel) for simplicity. To move along the $a / b$-axis, $\mathrm{He}$ (or $\mathrm{Ne}$ ) atoms have to pass through a rhombic 
(Rhom.) or rectangular (Rect.) gap as shown on Figure 2B'. The former is formed by the 291 intersection of $\mathrm{Zr}-\mathrm{Zr}$ and $\mathrm{O}-\mathrm{O}$ diagonals. The latter is formed by the intersection of $\mathrm{Si}-\mathrm{Si}$ and $\mathrm{O}-\mathrm{O}$ diagonals, and its area is much larger than the rhombic gap. Each channel consists of an alternating series of the two gap types (Figure 2B'); for example, along $a$ the sequence is S1-Rhom.-S1-Rect.-S1-Rhom.-S1-Rect. This configuration makes the a/b-channels considerably less diffusive than the $c$-axis due to the narrow rhombic gaps.

After channel determination, the two possible interstitial sites, called $S_{1}$ and $S_{1}$, were optimized. They are structurally symmetrical and energetically equivalent. Only one interstitial site environment exists for $\mathrm{He}$ and $\mathrm{Ne}$, an octahedral-like site formed by the six oxygen atoms.

299 The interatomic $\mathrm{He}-\mathrm{O}$ distances have three different values of 2.02, 2.22 and $2.36 \AA$. For the 300 Ne insertion case, the Ne atom is located 2.16, 2.30 and $2.42 \AA$ from neighboring oxygen atoms. 301 These larger distances are due to the larger size of the Ne atom, making the relaxation effect 302 important for $\mathrm{Ne}$.

The insertion energies calculated based on equation 1 for $\mathrm{He}$ and $\mathrm{Ne}$ in the different 304 insertion sites are listed in Table 1. The derived helium insertion energy is $1.46 \mathrm{eV}$, which is in 305 good agreement with other DFT calculations performed by Saadoune et al. (2009) and for 306 comparison obtained insertion value in hematite is given (Balout et al., 2017a, b). In the case 307 of $\mathrm{Ne}$, the insertion energy of $3.10 \mathrm{eV}$ is almost twice that calculated for He. This is related to 308 the larger atomic radius of $\mathrm{Ne}$, which must repel the neighboring atoms more to enter the 309 interstitial site.

\subsection{Migration Energies $\left(E_{m i g}\right)$}

In the $c$-channel, since the inter-site distance $S_{1}-S_{1}$ ' is about $1.5 \AA$, only one image is 312 taken between the two sites for the NEB calculation. Figure $3 \mathrm{~A}$ and $3 \mathrm{~A}^{\prime}$ present the migration 313 energies path results along the $c$-channel for $\mathrm{He}$ and $\mathrm{Ne}$ migration, respectively. The migration 314 energies, reported in Table 2, are $0.24 \mathrm{eV}$ for He (Figure 3A) and $0.22 \mathrm{eV}$ (Figure 3A') for the 
315 Ne atom. According to $a(b)$-channel structures, the $\mathrm{S}_{1}\left(\mathrm{~S}_{1}{ }^{\prime}\right)$ sites are separated on one side by

316 a rhombic gap and on the other by a rectangular one. Hence, two NEB calculations were 317 performed for $\mathrm{He}$ and Ne migration, corresponding to the energy necessary to pass through 318 each gap. For NEB calculation in this channel we start with a linear path composed of a series 319 of 4 images connecting the two $S_{1}\left(S_{1}{ }^{\prime}\right)$ sites. After the relaxation process we found that the 320 migration energy between the sites separated by the rectangular gap is equal to 0.66 and 1.53 $321 \mathrm{eV}$ for $\mathrm{He}$ and $\mathrm{Ne}$ atoms, respectively (Table 2; Figure 3B). For the rhombic gap, we find a migration energy of $2.60 \mathrm{eV}$ for $\mathrm{He}$, but for Ne the NEB calculation indicated that no migration is possible (Figure 3B'). Figure 4 illustrates the sequence of the interstitial sites along $c$ and how they are connected to the pathways along $a$ and $b$.

The paradoxical result is that along $c$ the energy barrier for $\mathrm{Ne}$ is lower than for $\mathrm{He}$, even though its radius is larger as already recognized in the insertion energy calculation. This can be understood by noting that the height of the barrier is a difference in energy. For Ne both the energy in the interstitial site and at the top of the barrier are higher in comparison to $\mathrm{He}$, reflecting the atom radius effect, but this does not say anything about the difference. As a conclusion, contrary to the insertion energy, the activation energy is not well controlled by the ionic or atomic radius. This outcome was already evident in Farley (2007). According to our results, one can say that, at low temperature, in an ideal crystal He and Ne will diffuse mainly along the $c$-channel. However, as temperature increases, there will be competition between the probability of jumping the $0.24 \mathrm{eV}$ barrier and the $0.66 \mathrm{eV}$ barrier in the case of He diffusion. For Ne-diffusion, however, even at high temperature diffusion along 336 the $c$-axis remains strongly favored.

\subsection{Ideal lattice results}

Using Transition State Theory as presented in section 2, the attempt frequencies $v$, which allow determination of relative jump probabilities, were calculated for each jump case. 
All calculated jump probabilities are provided in Table 3. From the parameters listed in this table, the diffusion coefficient along the $c$-axis can be derived analytically for a pristine crystal:

$$
D_{z}=v \cdot a^{2} \cdot \exp \left(-\frac{E_{m i g}}{k_{B} T}\right)
$$

343

with $a=1.5 \AA$. In the same way the diffusivity along $a$ and $b$ for an undamaged crystal and for practical temperatures $\left(\mathrm{T}<10^{4} \mathrm{~K}\right)$ is obtained as:

$$
D_{x y}=\frac{v \cdot a^{2}}{4} \cdot \exp \left(-\frac{E_{r e c t}}{k_{B} T}\right)
$$

with $a=3.35 \AA$. The factor 4 accounts for the fact that only one site over four allows a jump in a given direction (Fig. 4), as crossing the rhombic diamond is quasi-forbidden.

We get respectively for $\mathrm{He}$ and $\mathrm{Ne}$ :

$$
\begin{aligned}
& D_{z, H e}=1.6 \times 10^{-7} \exp \left(-\frac{0.24}{k_{B} T}\right) \mathrm{m}^{2} / \mathrm{s} \\
& D_{x y, H e}=1.5 \times 10^{-7} \exp \left(-\frac{0.66}{k_{B} T}\right) \mathrm{m}^{2} / \mathrm{s}
\end{aligned}
$$

$$
D_{x y, N e}=1.1 \times 10^{-7} \exp \left(-\frac{1.53}{k_{B} T}\right) \mathrm{m}^{2} / \mathrm{s}
$$

From these results one can conclude that $\mathrm{Ne}$ diffusivity is slightly higher at low temperature than He diffusivity in a perfect zircon host lattice. In addition, the diffusivity is highly anisotropic and diffusion occurs essentially along the $c$-axis. The anisotropy is even stronger in the Ne case due to the high energy barrier when crossing the rectangular gap.

\section{Discussion}

\subsection{He and Ne diffusion in non-damaged zircon structure}

\subsubsection{Comparison to previous calculations}

Helium diffusivity in zircon, calculated by DFT and molecular dynamics, has already been reported (Bengtson et al., 2012; Reich et al., 2007; Saadoune et al., 2009), and for 
comparison the insertion energy and barriers are listed respectively in Tables 1 and 2. It is

364 interesting to point out that only Bengtson et al. (2012) recognizes the $1.5 \AA$ inter-site distance 365 along the $c$-axis, which results from the crystal symmetries. Reich et al. (2007) and Saadoune et al. (2009) missed this feature although a dip is visible in the energy landscape for the former,

367 indicating that the minimal energy path was not found along the $c$-axis. Concerning the 368 migration energies, which have the strongest impact on the diffusivity, we are in good agreement with Saadoune et al. (2009) along the $c$-axis, and at variance with the other calculations. Although we found the same energy pattern along this channel as Bengtson et al. (2012), our migration energy is lower. As the variations of the energy along this channel are small the computations of the barrier are very sensitive to the finding of the minimum energy path. When comparing to the previous calculations along the $a / b$ directions we find the same 374 barrier across the rhombic gaps, but a higher one through the rectangular gap (0.66 instead of $\sim 0.45 \mathrm{eV}$, Figure 4, Table 2), for a reason which is not elucidated. We will see later that this is barrier is the one that mainly controls the diffusivity in zircon in practical situations. Concerning He diffusivity, differences exist with respect to the other calculations beyond the migration energy. Reich et al. (2007) and Saadoune et al. (2009) used a 1D formula which is known to be inadequate because it assumes that the energetic shape around the interstitial site and the saddle-point is the same. However, this assumption only acts on the preexponential factor and its impact is limited. Bengtson et al. (2012) did not use this assumption but assumed that along $a / b$ the higher barrier $(\sim 2.6 \mathrm{eV})$ dominates, disregarding the relatively easy intermediate jump along $c$ that can bypass this barrier. This explains why they obtained a 384 very low diffusivity along $a / b$.

\subsubsection{Comparison to experimental data}

For Ne only one abstract reports diffusion data, with an $E_{a}$ value of $\sim 339 \mathrm{~kJ} / \mathrm{mol}$ and 
value is much higher than that computed in this work. Similarly, He diffusion data obtained on natural zircon crystals present significantly higher apparent activation energies than obtained by theoretical studies. In addition, measured (U-Th)/He and (U-Th)/Ne zircon ages confirm that $\mathrm{He}$ and $\mathrm{Ne}$ are strongly retained in this mineral. For illustration, Figure 5 presents some $\mathrm{He}$ diffusion data obtained on natural zircon crystals (e.g., Reiners et al., 2004; Cherniak et al., 2009; Guenthner et al., 2013) compared to our data for a pristine zircon. In detail, Figure 5 shows the distribution of activation energy, frequency factor and closure temperature for He obtained for natural zircon, and the deduced activation energy for $\mathrm{Ne}$ needed to explain a (UTh)/Ne age (Gautheron et al., 2006). The authors based their estimate on the known thermal history of the Gold Butte, Nevada area (Reiners et al., 2000) and measured ZNe ages $(777 \pm 122$ and $963 \pm 164 \mathrm{Ma})$ that are much older than the ZHe age $(19.1 \pm 1.5$ and $16.7 \pm 1.3 \mathrm{Ma}$ respectively). From this, they deduced a closure temperature for $\mathrm{Ne}$ in zircon of $\sim 400 \pm 50^{\circ} \mathrm{C}$, similar to the diffusion data from Van Soest et al. (2013). The activation energy for $\mathrm{Ne}$ in zircon should thus be greater than the one for $\mathrm{He}$ (i.e., >170 kJ/mol), as Ne appears much more strongly retained than $\mathrm{He}$ (Figure 5), in contradiction with the result of our calculation in which a larger diffusivity is found along $c$. Based on the linear relationship between $E_{a}$ and closure temperature, for $\mathrm{Ne}$ we can anticipate an activation energy value above $\sim 170 \mathrm{~kJ} / \mathrm{mol}$, but we cannot clearly define the upper limit, and the lower limit is below the value determined by van Soest et al. (2013).

\subsection{Processes modifying $\mathrm{He}$ and Ne diffusion}

A strong discrepancy exists between results obtained using theoretical and experimental approaches, leading to a misinterpretation of the validity of the theoretical method. However, recent DFT studies on He and Ne diffusion in apatite (Djimbi et al., 2015) and hematite (Balout

411 et al., 2017a; b), which produce expectations consistent with experiments, bring a strong credit 412 to this theoretical approach. If the discrepancy is not due to a methodological issue, additional 
features and processes are likely to be modifying $\mathrm{He}$ and $\mathrm{Ne}$ diffusion in zircon, as already

414 discussed by Reich et al. (2007), Saadoune et al. (2009) and Bengtson et al. (2012).

Until now, our calculation has only considered an ideal pristine crystal, but in real

416 crystals defects are present and change local diffusion properties. The defects can be

417 substitutions in which an atom of the ideal lattice ( $\mathrm{Zr}$ usually) is exchanged for another, such

418 as a rare earth element. Another type is Frenkel pairs, consisting of an atom knocked out from

419 its original lattice site, leaving a vacancy, and inserted somewhere else. Frenkel pairs are

420 thermally activated and exist at any temperature, meaning that they can be created and

421 recombined by thermal motion. They are highly enhanced by irradiation due to the decay of $U$

422 and Th. The first effect of the alpha particle, and more strongly, the recoiling daughter is to

423 create Frenkel pairs by knocking atoms out of the lattice. For high radiation doses the density

424 of vacancies and interstitial atoms becomes high enough to make the lattice unstable, collapsing

425 it into amorphized zones. The diffusivity in such zones is not known and only hypotheses can

426 be proposed. Dislocations are yet another likely pervasive defect type that can alter diffusional

427 pathways. The detailed description of these defects is far beyond the scope of this work.

428 Similarly, meso-scale features that may alter diffusivity and retention, such as mineral and fluid

429 inclusions, are also beyond our scope. Our methodology is based on a simple modeling of their

430 effect on the diffusion of inserted $\mathrm{He}$ and $\mathrm{Ne}$ atoms, by considering that they diffuse in an ideal

431 crystal (Figure 6A) until they find some defect altering the diffusion (Figure 6B and 6C). We

432 address only two phenomena, obstruction and trapping.

433 Obstruction may occur when an interstitial atom, knocked out due to irradiation for

434 example, is already in an interstitial site, preventing $\mathrm{He}$ or $\mathrm{Ne}$ from entering the site. This

435 mechanism is efficient for slowing down diffusion along fast pathways along the $c$-axis in

436 zircon, because it requires the atom to jump over higher barriers to span long distances. The

437 motion is illustrated in Figure 6B, whereas Figure 6A shows the ideal case where the jumps 
occur primarily along the $c$-axis due to the low barrier. Obstruction can also result from 439 chemical substitutions that alter the energetics along the diffusion pathway. This has been 440 shown to occur for Cl-rich apatite compared to F-apatite (Djimbi et al., 2015), although the 441 authors demonstrate that a significant effect on diffusion only occurs at a relatively high 442 substitution level (some dozens of percent of the halogen sites) in that mineral. For zircon, Hf 443 substitution can be quite important (some wt\% percent, e.g., Hanchar and van Westrenen, 2007) 444 and will modify He diffusivity. Dislocations could also lead to obstruction by a local shrinking 445 of the original cell. In Figure 6B for example, the He atom first diffuses along the $c$-axis and crosses the rhombic gap but is then blocked due to the obstruction. Finally, the He atom will go back as it will demand too much energy to cross the rectangular gap, where the migration energy is $2.6 \mathrm{eV}(251 \mathrm{~kJ} / \mathrm{mol})$. As already emphasized in Djimbi et al. (2015), obstruction is very efficient in reducing diffusion anisotropy, which is an important feature in the case of zircon. The other effect we consider is trapping. This phenomenon has been already considered in several works (Farley, 2000; Shuster et al., 2006; Gautheron et al., 2009; Flowers et al., 2009; Shuster and Farley, 2009; Gerin et al., 2017) and can result from different kinds of defects. When vacancies are created, in particular under irradiation, they offer more space to the diffusing atom at lower energy. Therefore additional energy, noted $(\Delta \mathrm{Ea})$, is necessary to extract it from its location and bring it to a normal interstitial site. In general, trapping may occur in any situation where the interstitial atom has more space to be accommodated. The amorphized zones could offer this more open space and serve as He and Ne storage. If the zones 458 are not interconnected, diffusion still occurs through an ideal lattice endowed with trapping volumes. Even substitutions in some cases can act as traps. Saadoune and de Leeuw (2009) demonstrate that when $\mathrm{U}^{4+}$ and $\mathrm{Pu}^{4+}$ are present at the percent level in the zircon lattice in the 461 vicinity of an $\mathrm{He}$ insertion site, $\mathrm{U}$ and $\mathrm{Pu}$ increase $\mathrm{He}$ solubility by decreasing He insertion 462 energy, leading to a trapping effect. 
464 vacancies, and in the case of zircon amorphized zones (Figure 6C). This mechanism was initially proposed for apatite, where radiation damage strongly influences He diffusion by trapping He inside damage (Farley, 2000; Shuster et al., 2006). As a guideline, the maximum available trapping energy corresponds to a case where the diffusing atom is far from its neighbors, such as in a cavity (large damage, voids, fluid inclusions). Any short distance to its neighbors will repel it and increase its energy, thereby reducing $\left(\Delta E a_{T}\right)$. The extreme case where the atom is far from any other atom is equivalent to an atom outside of the crystal, and in this case the trapping energy is merely the insertion energy. Therefore, the maximal trapping energy is the insertion energy. The only exception to this rule would occur if atoms in the crystal would interact with the $\mathrm{He}$ or Ne by attracting it. Gerin et al. (2017) described the energetics of this process for apatite but similar conclusions can be drawn for zircon. As the insertion energy is 1.46 and $3.1 \mathrm{eV}$ for He and Ne respectively, one can infer that the maximal trapping energy for large damage such as recoil damage $\left(\Delta E a_{T}\right)$ will be respectively $1.46 \mathrm{eV}$ (with global $E a 1.7$ $\mathrm{eV}=1.46+0.24 \mathrm{eV} ; 164 \mathrm{~kJ} / \mathrm{mol}$ ) and $3.31 \mathrm{eV}$ (with global $E a 3.32 \mathrm{eV}=3.10+0.22 \mathrm{eV} ; 320$ $\mathrm{kJ} / \mathrm{mol}$ ). In crystals with real damage, different geometries of defects are present, entailing a distribution of $\Delta E a_{T}$. In addition, when radiation damage accumulates vacancies can connect creating larger spaces, increasing the trapping energy. Figure 6C illustrates a diffusion trajectory with different topologies of damage, with small damage (such as point defects), single recoil damage or recoil damage clusters. The $\Delta E a_{T}$ will be different for each case according to

483 the available free space. An important feature of trapping is that, unlike obstruction, it has no 484 effect on the anisotropy of diffusion, so that it cannot be responsible for the reduction of the anisotropy in case of zircon, although it has the capability to reduce diffusivity strongly, and may be spatially associated with other defects. 
It is expected that atoms displaced into the $c$-axis channel as a result of radiation damage strongly affects diffusion along $c$, which is quasi-free at normal temperature in an ideal lattice. In this section, we test the obstruction model using the KMC method and discuss the impact of obstructed pathways on $\mathrm{He}$ and $\mathrm{Ne}$ diffusion coefficients, by using the model described in section 2.3; additional details are given in Appendix A.

Figure 7 presents the dependence of the diffusion coefficient $D_{a, b}$ and $D_{c}$ (respectively along $a, b$ and $c$-axis) as a function of inverse temperature for a set of fractions of obstructed sites $(1,5,10,20 \%)$. We define the obstruction level as the fraction of sites occupied. In the hypothesis of obstructions produced by displaced atoms, the obstruction level is the fraction of displaced atoms multiplied by 1.5 , because there are 1.5 more atoms than interstitial sites $\mathrm{S} 1$ and S1'. Therefore, the considered set of obstruction levels corresponds respectively to $0.7,3.3$, 7, $13 \%$ of displaced atoms. Table 4 provides all obtained diffusion coefficients $\left(E a\right.$ and $\left.D_{0}\right)$ along the $a / b$-axis and $c$-axis and associated closure temperatures

Along $a$ - and $b$-channels the diffusion coefficient $\left(D_{a, b}\right.$ or $\left.D_{x, y}\right)$ is only slightly affected by the amount of obstruction (Figure 7A). The curves do not diverge significantly from each other; at most the $20 \%$ case reduces the diffusivity slightly, keeping the same slope defined by the barrier of $0.66 \mathrm{eV}$. Along the $c$-channel, obstruction strongly affects diffusivity $\left(D_{c}\right.$ or $\left.D_{z}\right)$, especially at low temperature where the slope of the diffusion behavior changes significantly (Figure 7B). An important result is that, surprisingly, the impact of obstruction on 3D diffusion is higher at low temperature with an important deviation from the ideal lattice. As the fraction 508 of obstruction increases the dependence moves closer to the $a / b$ diffusivity. For $\mathrm{T}<300^{\circ} \mathrm{C}$ all 509 the curves are almost straight lines parallel to the $a / b$ Arrhenius law, implying similar activation energy, but with $D_{0}$ scaling down as the level of obstruction increases. 
very strong in the ideal lattice, is reduced by a factor depending of the level of obstruction, but

514 almost independent of the temperature. For example, at $10 \%$ obstruction the anisotropy drops down to 20. It is worth noting that the above key trends are observed experimentally by

516 Guenthner et al. (2013), who show that He activation energy orthogonal to $c$ is only slightly 517 affected by damage, whereas with increasing damage diffusion along $c$ approaches a constant $518 E a$ close to that of the transverse direction. This experimental effect is reproduced by our 519 calculation. It reduces the anisotropy, compared to ideal crystal, and cannot be explained by a trapping effect alone. However, our modeled activation energy in any direction remains much smaller $(64 \mathrm{~kJ} / \mathrm{mol})$ in comparison to those measured in natural zircon (Reiners et al., 2004; Guenthner et al., 2013), which are around $169 \mathrm{~kJ} / \mathrm{mol}$. the primary parameter that drives retentivity of He in natural zircons. However, obstruction is the main parameter acting on the anisotropy of diffusion, reducing it strongly with respect to the ideal lattice case.

For Ne the change of diffusivity with obstruction is not progressive but total. At obstruction levels as low as $1 \%$, Ne diffusion is already frozen because the activation energy along the $a$ - and $b$-axes is so high $(147 \mathrm{~kJ} / \mathrm{mol}$, Table 3$)$. Nevertheless, as with He, diffusion

530 pathway obstruction alone cannot reproduce the inferred natural $\mathrm{Ne}$ diffusion behavior of 531 zircon; as described in section 4.2 , an activation energy $>180 \mathrm{~kJ} / \mathrm{mol}$ is necessary to reproduce

532 Ne ages (Gautheron et al., 2006), well above the maximum activation energy of $\sim 147 \mathrm{~kJ} / \mathrm{mol}$ 533 obtainable from obstruction. Thus, for $\mathrm{He}$ and $\mathrm{Ne}$, pathway obstruction mechanisms are not 534 sufficient to block He and Ne diffusion in zircon to the degree observed in natural specimens.

\subsection{Impact of traps on He and Ne diffusion in zircon}


the model published in Gerin et al. (2017) is used where the diffusion coefficient modified by damage, denoted $\widetilde{D}$, follows a formula (eq. 12) that includes a trapping term:

$$
\tilde{D}(x, t)=\frac{D(T)}{\left[1+f \times \exp \left(\frac{\Delta E_{a T}}{R T}\right)\right]}
$$

Where $D(T)$ is the diffusion coefficient of undamaged zircon (data from this study), $f$ is the damage fraction ranging from 0 to 1 , representing the normalized amount of displaced atoms in the structure, and $\Delta E_{a T}$ is the additional activation energy necessary for an $\mathrm{He}$ or $\mathrm{Ne}$ atom to jump out of the trap to the next insertion site. Appendix B provides a complete explanation and demonstration of the equation, and the conversion between damage fraction and alpha dose. In this study, the trapping model is correct only if damage stays as an isolated trap and does not form a percolating network connecting to the grain surface, as then it would not be a trap anymore.

The evolution of the simulated He closure temperature using Eq. 12 to account for equivalent alpha dose is reported in Figure $8 \mathrm{~A}$, for a $60-\mu \mathrm{m}$ radius spherical grain. The 551 simulation was done for $\Delta E_{a}$ values of $40,80,120$ and $140 \mathrm{~kJ} / \mathrm{mol}$, where $140 \mathrm{~kJ} / \mathrm{mol}$ is the maximum predicted value from the insertion energy $\left(\Delta E_{a T}\right)$ (see section 4.2). One can observe from Figure $8 \mathrm{~A}$ that a minimum $\Delta \mathrm{E}_{\mathrm{a}}$ of $120 \mathrm{~kJ} / \mathrm{mol}$ is necessary to reproduce retentive behavior in low-damage zircon of Guenthner et al. (2013). Interestingly, the activation energies and closure temperatures reported in Figure 8B are similar to those reported for natural samples in

556 Figure 5B, lending support to the idea that our calculations have reproduced the natural range 557 of trap diffusivities quite accurately. For $\mathrm{Ne}$, a similar simulation was done for the same $60-\mu \mathrm{m}$ 558 radius zircon, and results are reported in Figure $8 \mathrm{C}$ and D for $\Delta E_{a}$ of 160,200 and $300 \mathrm{~kJ} / \mathrm{mol}$, 559 as these values bracket the needed trapping to reproduce the inferred Ne closure temperature. 560 One can note that a trapping energy of $\geq 200 \mathrm{~kJ} / \mathrm{mol}$ is necessary to reproduce the inferred $\mathrm{Ne}$ 561 closure temperature. The data from van Soest et al. (2013) could not be used in this figure as 
562 the required information are not given in the conference paper. However, we note that the sum

563 of the insertion and migration energies we calculated for Ne corresponds to $320 \mathrm{~kJ} / \mathrm{mol}$, which

564 is comparable with the van Soest et al. (2013) value, and compatible with their Tc range if $\mathrm{D}_{0}$ 565 is adjusted.

566 For He, closure temperature will increase with the equivalent alpha-dose for relatively 567 low trapping energy $\Delta E a(<40 \mathrm{~kJ} / \mathrm{mol})$. It seems paradoxical that the closure temperature 568 depends only slightly on the damage fraction, as illustrated in Figure 8A, but this is well 569 understood as this fraction shows up in the pre-exponential factor $f$ in Eq. 12, whereas the 570 activation energy is in the exponential, giving rise to a much stronger effect. In other words 571 when the trapping energy is high most of the helium is trapped, whatever the number of traps 572 provided it is higher than the number of helium atoms, and only a high temperature (some 573 hundreds of ${ }^{\circ} \mathrm{C}$ during geological time) can extract them from the traps. This effect has been 574 seen with the DFT calculation in zircon (Saadoune and De Leeuw, 2009) and apatite (Gerin et 575 al., 2017), yet the damage topology between the two minerals is different. However, in the case 576 of zircon a higher trapping energy is needed to reproduce experimental data with respect to 577 expectations and calculations for apatite $(\sim 30-40 \mathrm{~kJ} / \mathrm{mol}$ for apatite (Gerin et al., 2017; Shuster 578 and Farley, 2009) compared to $120-140 \mathrm{~kJ} / \mathrm{mol}$ for zircon). The difference can be explained by 579 the higher insertion energy for $\mathrm{He}$ and even higher for $\mathrm{Ne}$ (Table 1) in zircon compared to 580 apatite ( 0.6 to $1 \mathrm{eV}$; Djimbi et al., 2015), as modification of the crystal structure during damage 581 creation will produce a site more energetically favorable compared to the lattice. In this case, 582 the insertion energy strongly drops, leading to an increase of the trapping energy until a 583 threshold $\Delta E_{a T}$. The higher necessary trapping energy can be explained by the damage topology 584 in zircon, as the crystal structure behaves quite differently than apatite. It is well known that 585 natural apatites are almost always crystalline in contrast to zircon, which is often reported to be 
partially or fully metamict (e.g. Ewing et al., 2003); additional infomation on damage can be

587 found in Appendix B.

\subsection{Damage percolation threshold}

For zircon, at high dose, several authors have reported that damage overlap or percolation may create amorphous zones (e.g. Ewing et al., 2003; Pidgeon, 2014; Salje et al., 1999). The more recent amorphous-crystal threshold has been estimated to be at $\sim 2.2 \times 10^{18} \mathrm{\alpha} / \mathrm{g}$ (Pidgeon, 2014), which has been interepreted as the recoil percolation damage threshold (Trachenko et al., 2003). The transition to rapidly rising diffusivity occurs at $\sim 2 \times 10^{18} \mathrm{\alpha} / \mathrm{g}$ in the diffusivity data set used by Guenthner et al. (2013), although data by Anderson et al. (2017) suggest a lower value at $2-5 \times 10^{17} \alpha / g$. In addition, Ketcham et al. (2013) calculated that alpha recoil and fission damage percolate at different thresholds than previously assumed $\left(\sim 2 \times 10^{16}\right.$ and $\sim 2 \times 10^{17} \alpha / g$ for alpha-recoil damage in 6-8-step decay chains and isolated decays (nochain) respectively, and $\sim 2 \times 10^{18} \alpha / g$ for fission tracks), due to the elongate nature of recoil and fission damage not taken into account by Trachenko et al. (2003).

Recent ZHe data measured in Hercynian granites from the Pyrenees (Bosch et al., 2016; Vacherat et al., 2016) exhibit age versus maximum alpha dose correlations that suggest that this retentivity maximum may be reached at $\sim 2-5 \times 10^{17} \alpha / \mathrm{g}$ (Figure 9). It is not straightforward to estimate the effective alpha dose needed to explain an age-eU pattern, so we have provided two solutions. First, as an end-member over-estimate, we simply retain all damage for each grain throughout its entire history since crystallization at $305 \mathrm{Ma}(\mathrm{U} / \mathrm{Pb}$ age); the dose can be no higher than this (Figure 9A). Second, we posit that the divergence of measured ages was due to a reheating and partial resetting event at some time in the past that caused more He loss in the less retentive grains, and so we should consider only the possible damage production prior to that event. A conservative estimate for its timing is the age of the youngest grain, leading to the 
relationship shown in Figure 9B. Even this dose is likely to be a significant over-estimate of

611 the pertinent dose for explaining the spread in ages, as it presumes that after crystallization 612 damage retention began immediately.

613 The implied transition dose from increasing to decreasing He retentivity is significantly 614 lower than to the one considered in the RDAAM model of Guenthner et al. (2013) and is closer 615 to the no-chain alpha recoil percolation level calculated by Ketcham et al. (2013). The no-chain 616 case is a possibly more appropriate approximation for connectivity that leads to fast pathways, as the connectivity due to $\mathrm{U}$ and Th decay chains is "tip to tip", and thus dominated by potentially obstructed regions at the boundaries of tracks. The dose range over which the nonchain percolating network expands to encompass the majority of recoil damage roughly coincides with the negative dose-age correlation in the Pyrenean samples, in the range 2 to

$6215 \times 10^{17} \alpha / g$, significantly below the dose range where the Guenthner et al. (2013) model 622 postulates a fall in retentivity (above $\sim 2 \times 10^{18} \alpha / g$ ). Such a lower alpha dose threshold for 623 percolation can also explain why samples in old and stable crustal blocks (Reiners et al., 2005) often displays only negative correlations between $\mathrm{ZHe}$ and $\mathrm{eU}$ (a proxy used for the alpha dose). In such cases the maximum potential retentivity that connects negative and positive correlations could not be documented because it would correspond to very low and atypical U 627 concentrations for zircon.

We infer from the Pyrenean samples that the recoil damage threshold will be at a value ranging around $\sim 2-5 \times 10^{17} \alpha / g$, as already proposed by Anderson et al. (2017), far below the $\sim 2 \times 10^{18} \alpha / g$ proposed by Guenthner et al. (2013).

\subsection{Implication for zircon $(U-T h) / H e$ and $(U-T h) / N e$ thermochronometry}


energetics, we can predict how He will diffuse in the zircon structure. Figure 10A provides a

636 schematic illustration of a diffusion pathway as postulated by our work. The He atom will 637 diffuse primarily along the $c$-axis following a random diffusion pathway. He diffusion is 638 facilitated along the $c$-axis because of the low migration energy; obstruction could slightly 639 change the trajectory but the He atom can diffuse easily along the $a$-axis to get around any 640 obstructed pathway. Following the example pathway of Figure 10A, the He atom is trapped in 641 a large defect (i.e. recoil damage, voids, fluid inclusion), with a very low insertion energy 642 (potentially approaching 0). The He atom will need a maximum activation energy of $1.7 \mathrm{eV}$ 643 (<164 kJ/mol) to go back to the crystal lattice (Fig. 10A). After that He diffuses again easily in 644 the lattice from site to site along the a-axis and $c$-axis (passage through the rhombic gap is 645 insignificant due to high migration energy, Fig. 4), until it reaches a second large energy well. 646 In this case the damage is connected to other damage extending to the crystal surface (Fig. 647 10A). In terms of energy, we can infer from our model that in the connected area, because the 648 lattice is distorted, the migration energy may be lowered. He insertion energy is thus very low 649 and the He atom will diffuse in the connected damage zone with lower energy barriers. In Figure $65010 \mathrm{~A}$, we predict that the He migration in the damage ranges from 1.4 to $0.9 \mathrm{eV}$ (135 to 87 $651 \mathrm{~kJ} / \mathrm{mol}$ ). Indeed, several geological studies show that for high damage dose, $\mathrm{ZHe}$ ages are similar to AFT ages (see Fig. 9 for the Pyrenean case example), and in some cases are even younger or equivalent to AHe ages (Johnson et al., 2017). This implies that at high dose (>10 18 $654 \alpha / g)$, He retentivity is low in damage zone, with Tc of 110 to $50^{\circ} \mathrm{C}$ and thus $E a$ of 135 to 90 $655 \mathrm{~kJ} / \mathrm{mol}$. We thus anticipate that, as opposed to the harmonic averaging model by Guenthner et al. (2013), diffusivity in zircon after the percolation transition may be controlled by the 657 energetics and topology of the damage network.

Figure 10B combines our modeling of trapping and obstruction with natural data in 659 terms of the closure temperature evolution as a function of the alpha dose. First, obstruction of 
diffusion pathways reduces diffusivity, not as efficiently as trapping but possibly enough to

661 play a role in addition to it. Indeed, at sufficient obstruction levels (> 20\% for He and $1 \%$ for

$662 \mathrm{Ne}$ ), diffusion for $\mathrm{He}$ and $\mathrm{Ne}$ will be only possible when it includes a component of motion

663 along the $a$ - and $b$-axis (Table 3). The resulting diffusion behavior will still not be retentive

664 enough to reproduce natural data, however. He and Ne global activation energy Ea values are 665 respectively $63 \mathrm{~kJ} / \mathrm{mol}$ and $147 \mathrm{~kJ} / \mathrm{mol}$, while values of $120-140 \mathrm{~kJ} / \mathrm{mol}$ and $>180 \mathrm{~kJ} / \mathrm{mol}$ are 666 needed (Figure 10B for He). The 20\% obstruction level corresponds to $13 \%$ of atoms displaced 667 (and so to an equivalent dose of $\sim 10^{18} \alpha / \mathrm{g}$ ) as explained in Appendix B, and corresponds to an 668 equivalent closure temperature of approximately $-75^{\circ} \mathrm{C}$ assuming a $\mathrm{D}_{0}$ of $1.5 \times 10^{-7} \mathrm{~m}^{2} / \mathrm{s}$. This 669 value is not very tightly constrained, but provides a reasonable, illustrative first estimate of He 670 retention.

If our modeling is correct, it is possible to make some predictions. First, for $\mathrm{He}$ and $\mathrm{Ne}$, 672 a quite high trapping energy is necessary to reproduce closure temperatures inferred from 673 natural samples (Figure 8 and Figure 10B), and we predict maximum possible trapping energies 674 with $\Delta \mathrm{EaT}_{\mathrm{T}}$ of $140 \mathrm{~kJ} / \mathrm{mol}\left(\mathrm{E}_{\mathrm{mig}}=1.46 \mathrm{eV}\right)$ and $300 \mathrm{~kJ} / \mathrm{mol}\left(\mathrm{E}_{\mathrm{mig}}=3.10 \mathrm{eV}\right)$ for $\mathrm{He}$ and $\mathrm{Ne}$ 675 respectively. These trapping energies $(\triangle \mathrm{EaT})$ are within the limits provided by our DFT 676 calculations, as discussed in section 4.2. It means that the total energy needed to go back in the 677 crystal structure will be $164 \mathrm{~kJ} / \mathrm{mol}\left(E_{\text {insertion }}+E_{\text {mig }}=1.7 \mathrm{eV}\right)$ and $320 \mathrm{~kJ} / \mathrm{mol}\left(E_{\text {insertion }}+\right.$ $678 E_{m i g}=3.32 \mathrm{eV}$ ) for $\mathrm{He}$ and Ne respectively. Second, based on published very young volcanic 679 zircon of ZHe ages coherent with other methods (e.g. Farley et al., 2002), we can infer that for 680 low alpha dose $\left(\sim 1-2 \times 10^{15} \alpha / \mathrm{g}\right)$, He is retentive to surface temperature implying a Tc of $\sim 60$ $681100^{\circ} \mathrm{C}$ (Fig. 10B). Between low damage and the threshold, we could anticipate that Tc may 682 increase following a non-linear (i.e. cubic or exponential) law as is the case for apatite (Flowers 683 et al., 2009), as $\Delta E a$ increases until the $\Delta E a_{T}$ threshold (Fig. 10B). As damage fraction 684 increases, clustering increases also and damaged domains with different sizes will form. In this 
case, the trapping energy can increase as proposed by Recanati et al. (2017) for apatite. It is interesting to note that the highest closure temperatures obtained by Guenthner et al. (2013) can be explained by increasing trapping energy when damage dose increases up to a maximum (Fig. 8A). For $\mathrm{Ne}$, the maximum activation energy predicted in this study is similarly very close to the one reported by van Soest et al. (2013), and we can anticipate a complex relationship of $\mathrm{Ne}$ diffusion with damage dose similar to He.

After some damage threshold, He diffusion increases (Tc decreases), although the Pyrenees samples (Bosch et al., 2016; Vacherat et al., 2016) and work of Johnson et al. (2017) indicate that the threshold dose may be lower than indicated by Guenthner et al. (2013). The

694 source of this discrepancy may be a complex function of damage accumulation and annealing, 695 and more work on this issue is essential. For example, Ginster et al. (2018) document a 696 complicated relationship between damage dose and annealing rate, implying a very complex 697 system.

The other implication for a maximum of retentivity located in the vicinity of the no699 chain alpha damage percolation level (Fig. 9) would be that the dependence of closure 700 temperature on damage fraction increase can be significantly steeper than modeled with Eq. 12 701 (Fig. 10B) if a single $\Delta E a_{T}$ value is assumed. This additional level of complexity will also have 702 implications for developing very precise future quantitative models to predict individual $\mathrm{T}_{\mathrm{C}}$ values for single zircon grains. Such a potential scenario supported by natural data will require

704 new experimental diffusion data to document carefully this key window of alpha-dose in the 705 range of the no-chain percolation level.

\section{5. Conclusions}

In this contribution, we have used a series of computational and theoretical tools to 708 provide a mechanistic view of the manner in which He and Ne diffuse in zircon. In particular, 709 we have characterized the undamaged crystalline state and its energy structure at the atomic 
scale with respect to the diffusing atoms, and how trapping and obstruction as may result from

711 damage or inherent defects affect retention at the grain scale. The set of insights gained can 712 help leverage ongoing analytical efforts to unravel the complex behavior of the (U-Th)/He and

713 (U-Th)/Ne systems in natural settings. Using Density Functional Theory and Transition State theory, we have calculated the insertion energies for $\mathrm{He}$ and $\mathrm{Ne}$ in ideal zircon, as well as the migration pathways along the $a / b$ and $c$ axes and the migration energies for each jump. For diffusion in the $a / b$ direction, we document the energetics of the rectangular and rhombic pathways to neighboring interstitial sites. Our insertion energies for $\mathrm{He}$ are similar to previous work, but our migration energies and their interrelationships are different; notably, our work finds that the relative migration energies along the $c$-axis channel and through the rectangular and rhombic $a / b$ gaps are approximately 0.24 vs. 0.66 and $2.60 \mathrm{eV}$ for $\mathrm{He}$, and 0.22 vs. $1.53 \mathrm{eV}$ and infinite for Ne. One important finding is that our insertion energies, in combination with our migration energies, predict the maximum activation energies possible for the zircon $\mathrm{He}$ and $\mathrm{Ne}$ systems, which are consistent with literature values.

Our results corroborate previous theoretical and analytical work implying that pristine zircon is non-retentive for He due to low migration energies along the $c$-axis channels in the crystal structure. We also obtain the unexpected result that Ne moves even more freely than He through an unblocked $c$-axis channel, due to the distortion it causes in the insertion site. Both systems therefore require additional factors to explain their natural behavior. Using the Kinetic Monte Carlo method we show that obstruction of $c$-axis pathways forces the migration energetics to become more similar to the slower $a / b$ direction as blockages increase, causing diffusion to become effectively isotropic on the grain scale and characterized by rectangularpath-jump energetics. This effect is insufficient to explain slow diffusion of He in zircon, but

734 has a larger impact on $\mathrm{Ne}$, as rectangular $a / b$ transitions are much higher-energy, and rhombic 
ones are impassable. Of the mechanisms we inspect, we find that trapping is required to explain the high retention of $\mathrm{He}$ and $\mathrm{Ne}$ observed in natural zircon.

We consider the topology of radiation damage in the context of a data set from Pyrenees granites that indicates a transition from falling to rising diffusivity at a much smaller dose than posed in the Guenthner et al. (2013) diffusivity model, closer to $\sim 2-5 \times 10^{17}$ than $2 \times 10^{18} \alpha / \mathrm{g}$. The transition dose may correspond to the no-chain alpha recoil percolation threshold of Ketcham et al. (2013), suggesting that tip-to-tip recoil track connectivity is not sufficient to form fast diffusion pathways, but more interpenetrative damage intersections can. Increasing track intersections may also create more and deeper traps, and the evolution of not just trap abundance but also trap energetics may be necessary to arrive at a quantitative understanding of rare gas diffusion in natural zircon.

\section{Acknowledgment}

This project and salary for Duval Mbongo-Djimbi were funded by the Agence National de la Recherche - grant no. ANR-12-BS06-0005-01. DIM OXYMORE funded by the region île de France is thanked for salary for Hilal Balout. We thank Christophe Diarra for his precious help in managing the IPNO cluster GRIF (http:// www.grif.fr). William Guenthner is warmly thanked for sharing the diffusion data of his 2013 contribution. Daniele Cherniak, Peter Reiners and Martin Danišík are warmly thanked for their constructive reviews.

\section{Appendix A and B - Supplementary data}

Supplementary data associated with this article can be found, in the online version. 


\section{References}

756 Anderson A.J., Hodges K.V. and van Soest M.C. (2017) Empirical constraints on the effects of radiation damage on helium diffusion in zircon. Geochim. Cosmoch. Acta. 218, 308322.

Balout H., Roques J., Gautheron C., Tassan-Got L. and Mbongo-Djimbi D. (2017a) Helium diffusion in pure hematite $\left(\alpha-\mathrm{Fe}_{2} \mathrm{O}_{3}\right)$ for thermochronometric applications: a theoretical multi-scale study. Computational and Theoritical Chemistry, 1099, 21-28.

762

763

764

765

766

767

768

769

770

771

772

773

774

775

Balout H., Roques J., Gautheron C. and Tassan-Got, L. (2017b) Computational investigation of the interstitial neon diffusion in pure hematite, $\alpha-\mathrm{Fe}_{2} \mathrm{O}_{3}$. Computational Materials Science, 128, 67-74.

Bengtson A., Ewing R.C. and Becker U. (2012) He diffusion and closure temperatures in apatite and zircon: A density functional theory investigation. Geochim. Cosmoch. Acta, 86, $228-238$.

Bortz A.B., Kalos M.H. and Lebowitz J.L. (1975) A new algorithm for Monte Carlo simulation of Ising spin systems. J. Comput. Phys., 17, 10-18.

Bosch V., Teixell G.A., Jolivet M., Labaume P., Stockli D., Domènech M. and Monié P. (2016) Timing of Eocene-Miocene thrust activity in the Western Axial Zone and Chaînons Béarnais (west-central Pyrenees) revealed by multi-method thermochronology. Comptes Rendus Geoscience, 348, 246-256.

Cherniak D.J., Watson E.B. and Thomas J.B. (2009) Diffusion of helium in zircon and apatite. Chem. Geol., 268, 155-166.

Danišík, M., McInnes, B.I.A., Kirkland, C.L., McDonald, B.J., Evans, J.P. and Becker, T. (2017) Seeing is believing: Visualization of He distribution in zircon and implications for thermal history reconstruction on single crystals. Sci. Adv. 3. e1601121 
Djimbi D.M., Gautheron C., Roques J., Tassan-Got L., Gerin C. and Simoni E. (2015) Impact of apatite chemical composition on (U-Th)/He thermochronometry: an atomistic point of view. Geochim. Cosmoch. Acta, 167, 162-176.

782

Dodson M.H. (1973) Closure temperature in cooling geochronological and petrological systems. Contrib. Min. Petrol., 40, 259-274.

Elber R. and Karplus M. (1987) A method for determining reaction paths in large molecules: Application to myoglobin. Chem. Phys. Lett. 139, 375-380.

Ewing R.C., Meldrum A., Wang L.M., Weber W.J. and Corrales L.R. (2003). Radiation damage in zircon. In: Hanchar, J.M., Hoskin, P.W.O. (Ed.), Zircon. Mineralogical Society of America, pp. 387-425.

Farley, K.A. (2000) Helium diffusion from apatite: general behavior as illustrated by Durango fluorapatite. J. Geophys. Res. 105, 2903-2914.

Farley K.A. (2007) He diffusion systematics in minerals: Evidence from synthetic monazite and zircon structure phosphates. Geochim. Cosmoch. Acta. 71, 4015-4024.

Farley K.A. and Flowers R. (2012) (U-Th)/Ne and multidomain (U-Th)/He systematics of a hydrothermal hematite from Eastern Grand Canyon. Earth Planet. Sci. Lett., 359-360, 131-140.

Farley K.A. and Stockli D.F. (2002) (U-Th)/He Dating of Phosphates: Apatite, Monazite, and Xenotime., Reviews in Mineralogy and Geochemistry, pp. 559-577.

Flowers R., Ketcham R.A., Shuster D. and Farley K.A. (2009) Apatite (U-Th)/He thermochronology using a radiation damage accumulation and annealing model. Geochim. Cosmochim. Acta 73, 2347-2365.

Gautheron C. and Tassan-Got L. (2010) A Monte Carlo approach of diffusion applied to noble gas/helium thermochronology. Chem. Geol. 273, 212-224. 
803 Gautheron C., Tassan-Got L. and Farley K.A. (2006) (U-Th)/Ne chronometry. Earth Planet.

804

805

806

807

808

809

810

811

812

813

814

815

816

817

818

819

820

821

822

823

824

825

826 Sci. Lett. 243, 520-535.

Gautheron C., Tassan-Got L., Ketcham R.A. and Dobson K.J. (2012) Accounting for long alpha-particle stopping distances in (U-Th-Sm)/He geochronology: 3D modeling of diffusion, zoning, implantation, and abrasion. Geochim. Cosmochim. Acta 96, 44-56.

Gautheron C.E., Tassan-Got L., Barbarand J. and Pagel M. (2009) Effect of alpha-damage annealing on apatite (U-Th)/He thermochronology. Chem. Geol. 266, 166-179.

Gerin C., Gautheron C., Oliviero E., Bachelet C., Djimbi M.D., Seydoux-Guillaume A.M., Tassan-Got L., Sarda P., Roques J. and Garrido F. (2017) Influence of vacancy damage on He diffusion in apatite investigated at atomic to mineralogical scales. Geochim. Cosmochim. Acta 197, 87-103.

Ginster, U., Reiners, P. W., Nasdala, L., and Chanmuang, C. N., 2019, Annealing kinetics of radiation damage in zircon: Geochim. Cosmoch. Acta. 249, 225-246.

Guenthner W., Reiners P.W., Ketcham R., Nasdala L. and Giester G. (2013) Helium diffusion in natural zircon: radiation damage, anisotropy, and the interpretation of zircon (UTh)/He thermochronology. Am. J. Sci. 313, 145-198.

Hanchar, J. M., and van Westrenen, W., 2007, Rare Earth element behavior in zircon-melt systems. Elements, 3, 37-42.

Johnson, J.E., Flowers, R.M., Baird, G.B. and Maham, K.M. (2017) "Inverted” zircon and apatite (U-Th)/He dates from the Front Range, Colorado: High-damage zircon as a lowtemperature $\left(<50^{\circ} \mathrm{C}\right)$ thermochronometer. Earth Planet. Sci. Lett. 466, 80-90.

Jónsson H., Mills G. and Jacobsen K.W. (1998) Nudged Elastic Band Method for Finding Minimum Energy Paths of Transitions. Classical and Quantum Dynamics in Condensed Phase Simulations 385 pp. 
Ketcham R.A., Guenthner W.R. annd Reiners P.W. (2013) Geometric analysis of radiation damage connectivity in zircon, and its implications for helium diffusion. Am. Mineral. 98, 350-360.

Kohn W. and Sham L.J. (1965) Self-Consistent Equations Including Exchange and Correlation Effects. Phys. Rev. 140, A1133-A1138.

Kresse G. and Furthmüller J. (1996) Efficient iterative schemes for ab initio total-energy calculations using a plane-wave basis set. Phys. Rev. B. 54, 11169.

Kresse G. and Hafner J. (1993) Ab initio molecular dynamics for liquid metals. Phys. Rev. B. 47, 558(R).

Mills G., Jónsson H. and Schenter G.K. (1995) Reversible work transition state theory: application to dissociative adsorption of hydrogen. Surf. Sci. 324, 305-337.

Pratt L.R. (1986) A statistical method for identifying transition states in high dimensional problems. J. Chem. Phys. 85, 5045-5048.

Recanati A., Gautheron C., Barbarand J., Missenard Y., Pinna-Jamme R., Tassan-Got L., Carter A., Douville E., Bordier L., Pagel M. and Gallagher K. (2017) Helium trapping in apatite damage: Insights from (U-Th-Sm)/He dating of different granitoid lithologies. Chem. Geol. 470, 116-131.

Reich M., Ewing R.C., Ehlers T. and Becker U. (2007) Low-temperature anisotropic diffusion of helium in zircon: Implications for zircon (U-Th)/He thermochronometry. Geochim. Cosmochim. Acta 71, 3119-3130.

Reiners P.W. (2005) Zircon (U-Th)/He thermochronometry. In: Reiners, P.W., Ehlers, T.A. (Eds.), Thermochronology, Rev. Mineral. Geochem. pp. 151-179.

Reiners P.W., Brady R., Farley K.A., Fryxell J.E., Wernicke B.P. and Lux D. (2000) Helium and argon thermochronology of the Gold Butte block, south Virgin Mountains, Nevada. Earth Planet. Sci. Lett. 178, 315-326. 
852 Reiners P.W., Farley K.A. and Hiskes H.J. (2002) He diffusion and (U-Th)/He

853

854

855

856

857

858

860

861

862

863

864

865

866

867

868

869

870

871

872

873

874 thermochronology of zircon: initial results from Fish Canyon Tuff and Gold Butte. Tectonophysics 349, 297-308.

Reiners P.W., Spell T.L., Nicolescu S. and Zanetti K.A. (2004) Zircon (U-Th)/He thermochronometry: He diffusion and comparison with ${ }^{40} \mathrm{Ar} /{ }^{39} \mathrm{Ar}$ dating. Geochim. Cosmochim. Acta 68, 1857-1887.

Saadoune I. and de Leeuw N.H. (2009) A computer simulation study of the accomodation and diffusion of $\mathrm{He}$ in uranium- and plutonium-doped zircon $\left(\mathrm{ZrSiO}_{4}\right)$. Geochim. Cosmochim. Acta 73, 3880-3893.

Saadoune I., Purton J.A. and de Leeuw N.H. (2009) He incorporation and diffusion pathways in pure and defective zircon $\mathrm{ZrSiO}_{4}$ : A density functional theory study. Chem. Geol. 258, 182-196.

Salje E.K.H., Chrosch J. and Ewing R.C. (1999) Is "metamictization" of zircon a phase transition? Am. Mineral. 84, 1107-1116.

Shuster D. and Farley K.A. (2009) The influence of artificial radiation damage and thermal annealing on helium diffusion kinetics in apatite. Geochim. Cosmochim. Acta 73, 183196.

Shuster D., Flowers R. and Farley K.A. (2006) The influence of natural radiation damage on helium diffusion kinetics in apatite. Earth Planet. Sci. Lett. 249, 148-161.

Shuster D., Vasconcelos P., Heim J. and Farley K.A. (2005) Weathering geochronology by (UTh)/He dating of goethite. Geochim. Cosmochim. Acta 69, 659-673.

Trachenko K., Dove M.T. and Salje E.K. (2003) Large swelling and percolation in irradiated zircon. J. Phys. Condens. Matter 15, L1-L7. 
875 Vacherat A., Mouthereau F., Pik R., Bellahsen N., Gautheron C., Bernet M., Daudet M., Balansa J., Tibari B., Pinna-Jamme R. and Radal J. (2016) Rift-to-collision transition recorded by tectono-thermal evolution of the northern Pyrenees. Tectonics 35, 4 .

van Soest M.C., Tripathy-Lang A., Hodge K.V. and Monteleone B.D. (2013) Neon diffusion in titanite and zircon and the utility of these minerals for (U-Th)/Ne thermochronometry. In: AGU (Editor), American Geophysical Union, Fall Meeting. AGU, San Francisco.

Vineyard G.H. (1957) Frequency factors and isotope effects in solid state rate processes. $J$. Phys. Chem. Solids 3, 121-127.

Wert C. and Zener C. (1949) Interstitial Atomic Diffusion Coefficients. Phys. Rev. 76, 11691175.

Zeitler P.K., Herczerg A.L., McDougall I. and Honda M. (1987) U-Th-He dating of apatite: A 886 potential thermochronometer. Geochim. Cosmochim. Acta 51, 2865-2868. 
892

893 Table 1: Helium and neon insertion energies in zircon, hematite and apatite crystal structure.

894

895 Table 2: Migration energies in $\mathrm{eV}$ for both helium and neon in zircon compared with the 896 theoretical studies of He diffusion (Bengtson et al., 2012; Reich et al., 2007; Saadoune et al., 897 2009). Rect. and Rhom. are for the rectangular and rhombic gap.

898

899 Table 3: Diffusion parameters for $\mathrm{He}$ and $\mathrm{Ne}$ in ideal lattice.

900

901 Table 4: Diffusion coefficients $\left(\mathrm{D}_{0}\right.$ and $\left.\mathrm{Ea}\right)$ along $a / b$ and $c$ axes for different percentages of 902 obstruction, as shown in Figure 8.

903

904 
Figure 1: Sequence of computational tools developed and used in this study. Migration

908

909

910

911

912

913

914

915

916

917

918

919

920

921

922

923

924

925

926

927

928

929

Figure 2: Representation of the zircon crystal supercell $(2 \times 2 \times 2)$ showing the channels along the $c$-axis (A) and along the $a$ - and $b$-axes (B). He/Ne insertion sites indicated by square and circle. Along the diffusion $c$-channel and $a$ - and $b$-channel (note that the $a$ - and $b$-axes are identical), the different $\mathrm{He} / \mathrm{Ne}$ insertion sites $\mathrm{S}_{1}$ and $\mathrm{S}_{1}$ ' are denoted (A' and B'). Along $a$ - and b-channels, $\mathrm{He} / \mathrm{Ne}$ atoms have to pass through a rhombic (Rhom.) or rectangular (Rect.) gap. Note that along the $a$-axis the sites are only S1 type whereas along $b$-axis the sites are only S1' type.

Figure 3: Evolution of the minimal energy path (MEP) between insertion site $S_{1}$ to $S_{1}$ ' along the $c$-channel (A) and (A') and along $a$ - and $b$ - channels (B) and (B') for He and $\mathrm{Ne}$ respectively. Rect. and Rhom. are for the rectangular and rhombic gaps. As with Figure 2, the sites along the $a$ and $b$-axes are $\mathrm{S} 1$ and $\mathrm{S} 1$ ' respectively. 
Figure 4: Schematic illustration of the different diffusion pathways in zircon identified with the insertion energies along the three axes $(a, b$ and $c)$. The two sites $\mathrm{S}_{1}$ and $\mathrm{S}_{1}$ ' are distinguished and the example of different diffusion pathways between those sites are shown.

Figure 5: Correlation of the activation energy $E_{a}$ with frequency factor $\mathrm{D}_{0}(\mathrm{~A})$ and closure temperature (B) determined in 3D for $\mathrm{He}$ and $\mathrm{Ne}$ (green diamonds). Results obtained on natural zircon crystal by Cherniak et al. (2009); Guenthner et al. (2013); Reiners et al. (2004) for $\mathrm{He}$ and are reported. A minimal deduced $E_{a}$ value for $\mathrm{Ne}$ obtained from the closure temperature estimate of Gautheron et al. (2006) is reported in diagram B.

Figure 6: Schematic representation of mechanisms that may affect diffusivity. (A) He and Ne diffusion pathway depends only on diffusion coefficients between all insertion sites. $E a_{c}, E a_{r h o m}$ and $E a_{r e c t}$ refer to the activation energies along the $c$-axis and through the $a$-axis

944 rhombic and rectangular gaps. The same color codes as in Figure 4 are used for diffusion 945 through rectangular or rhombic gaps. One can note that the energy to diffuse through a rhombic 946 gap is high compare to a rectangular one, and that no diffusion can occur through impassable 947 boundaries (thick black lines). (B) Some pathways are obstructed (blue cross), and $\mathrm{He}$ or $\mathrm{Ne}$ 948 can only go around the blocked paths. Same diffusion coefficient between insertion sites is 949 taken as in A. (C) Damage represented by the open blue oval can trap atoms, as the needed $950 \Delta E a_{T}$ is much higher than the activation energy between interstitial sites.

Figure 7: Evolution of the diffusion coefficient as a function of inverse temperature 953 along $a / b$-axis (A) and $c$-axis (B) for various values of obstructed pathways from 1 to $20 \%$. As 954 a reference the diffusion coefficient evolution in 3D and for all axes, and for natural zircon 
955 (gray line; Reiners et al., 2004; orange lines with similar dashed lines from Guenthner et al., 956 2013) are reported in both figures.

957

958

959

960

961

962

963

964

965

966

967

968

969

970

971

972

973

974

975

976

977

978

979

Figure 8: Models of helium and neon closure temperature dependence on alpha dose $(\mathrm{A}, \mathrm{C})$ and activation energy (B, D). Closure temperature evolution with dose (A, C, black lines) modeled for a $60-\mu \mathrm{m}$ radius spherical grain, using the base level diffusion coefficients obtained in this study and equation (12) to estimate the effects of dose and trapping energy. $\Delta E a$ values of $40,80,100,120$ and $\Delta E a_{T}$ of $140 \mathrm{~kJ} / \mathrm{mol}$ are modeled for He, and $\Delta E a$ values of 160,200 and $\Delta E a_{T}$ of $300 \mathrm{~kJ} / \mathrm{mol}$ for Ne. Diagram $\mathrm{B}$ and $\mathrm{D}$ shows the evolution of the modified activation energy as a function of the calculated closure temperature. Data obtained from this study (diamonds), on natural zircon from Guenthner et al. (2013) (red dots; Figures A, B), and data from van Soest et al. (2013) for Ne diffusion are reported for comparison. Black and gray circles same as in Figure 5.

Figure 9: ZHe age evolution as a function of the equivalent alpha-dose, for two Pyrenean massifs (Bosch et al., 2016; Vacherat et al., 2016). The values for the 6-8-chain, nochain and fission-track percolation thresholds of Ketcham et al. (2013) are reported for comparison. Apatite fission track (AFT) and apatite (U-Th)/He (AHe) ages range from Bosch et al. (2016) and Vacherat et al. (2016) are also shown for comparison.

Figure 10: (A) Schematic representation of the energetic levels of a diffusion pathway for He; a similar representation can be made for $\mathrm{Ne} .1 .46 \mathrm{eV}$ is the He insertion energy, and $\mathrm{E}_{\mathrm{mig}}$ is the migration energy, that can be for $\mathrm{He} 0.24,0.66 \mathrm{eV}$ for regular lattice (24 and 66 $\mathrm{kJ} / \mathrm{mol}$ respectively). In a damage zone, He insertion can be lower and the resulting trapping energy will increase and can be up to $1.46 \mathrm{eV}$, resulting in a migration energy of $1.7 \mathrm{eV}$ 
980 ( $\mathrm{E}_{\mathrm{mig} .}=164 \mathrm{~kJ} / \mathrm{mol}$ ). In this model, the size of the regular insertion site (aIs, black arrow) is 981 smaller than for a damage zone (aD, blue arrow) resulting in a different energy level. (B) 982 Evolution of the closure temperature as a function of the alpha dose (black lines). Data from 983 Guenthner et al. (2013) (red circles) and calculated with the DFT results (this study, red square) 984 are reported in addition to the trapping model with trapping energies $\Delta \mathrm{Ea}$ of 100,120 and $\Delta \mathrm{EaT}$ $985140 \mathrm{~kJ} / \mathrm{mol}$, and the blocking pathway model at 1, 5, 10 and $20 \%$ of obstruction (details about 986 the calculation are given in Table 4). The calculated damage thresholds from Ketcham et al. 987 (2013) and the one inferred from Pyrenees samples (Fig. 9) are also reported. Inferred closure 988 temperature for very young zircon (<1 Ma; Farley et al., 2002), Pyrenean samples (Bosch et al., 989 2016; Vacherat et al., 2016) and highly damages zircon from Johnson et al. (2017) are also 990 shown. The relation between obstructed pathways with alpha dose is not straightforward, but 991 we estimate the transition from rising to falling closure temperature to be in the $2-5 \times 10^{17} \alpha / \mathrm{g}$ 992 range.

993 
Table 1. Insertion energies for He and Ne into interstitial sites in this and other studies.

\begin{tabular}{ccccc}
\hline $\begin{array}{c}\text { Insertion } \\
\text { energy }\end{array}$ & $\begin{array}{c}\text { Zircon, this } \\
\text { work }\end{array}$ & $\begin{array}{c}\text { Zircon, Saadoune } \\
\text { et al. (2009) }\end{array}$ & $\begin{array}{c}\text { Hematite, Balout et } \\
\text { al. (2017a, b) }\end{array}$ & $\begin{array}{c}\text { Apatite, Djimbi } \\
\text { et al. (2015) }\end{array}$ \\
\hline $\mathrm{He}(\mathrm{eV})$ & 1.46 & 1.45 & 1.51 & 1.15 (a,b-plan); \\
& & & & 0.66 (c-axis) \\
$\mathrm{Ne}(\mathrm{eV})$ & 3.10 & - & 3.38 & - \\
\hline
\end{tabular}


Table 2. Migration energies (in $\mathrm{eV}$ ) for $\mathrm{He}$ and $\mathrm{Ne}$ in zircon.

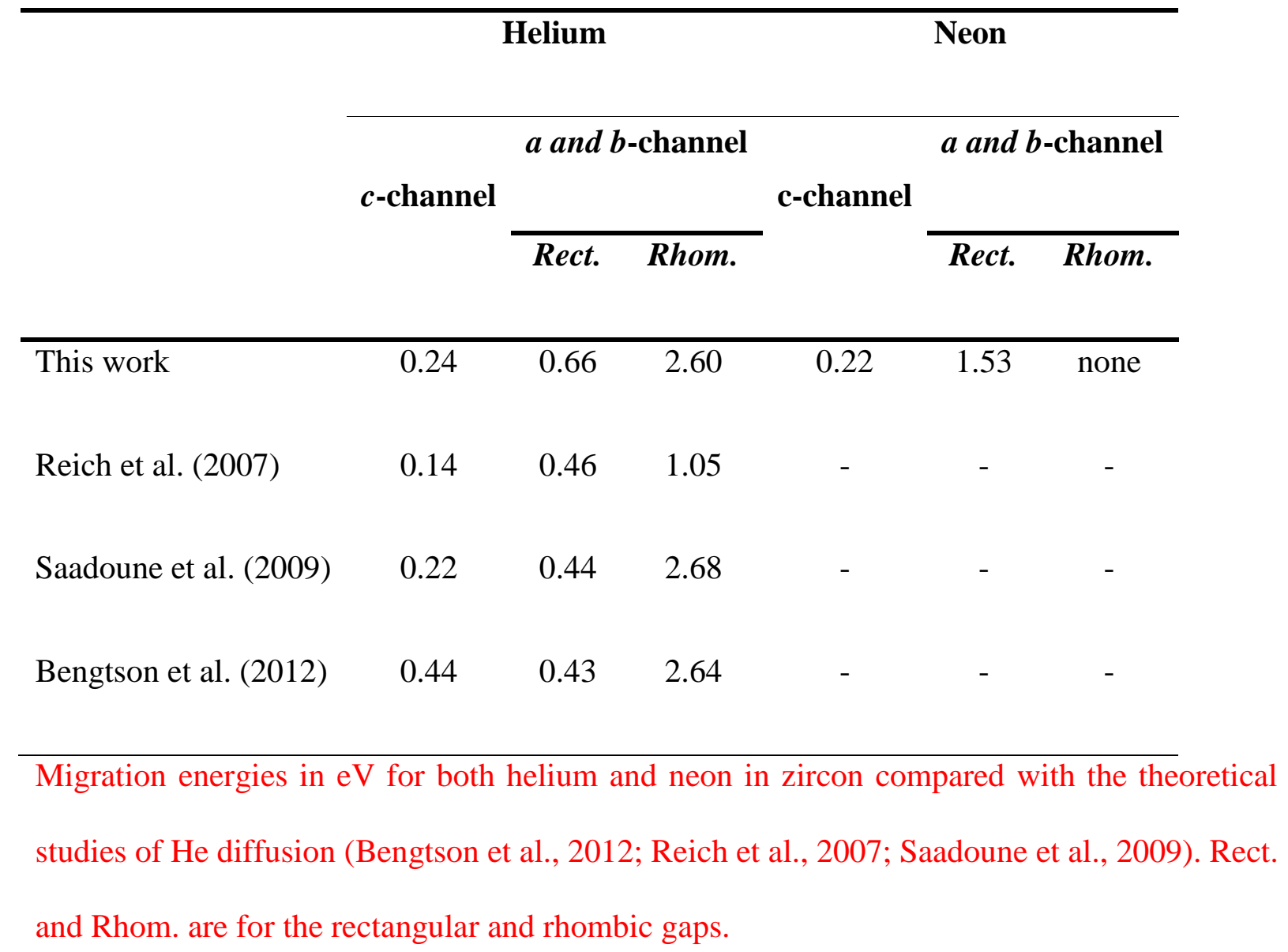


Table 3. Diffusion parameters for He and Ne in ideal lattice.

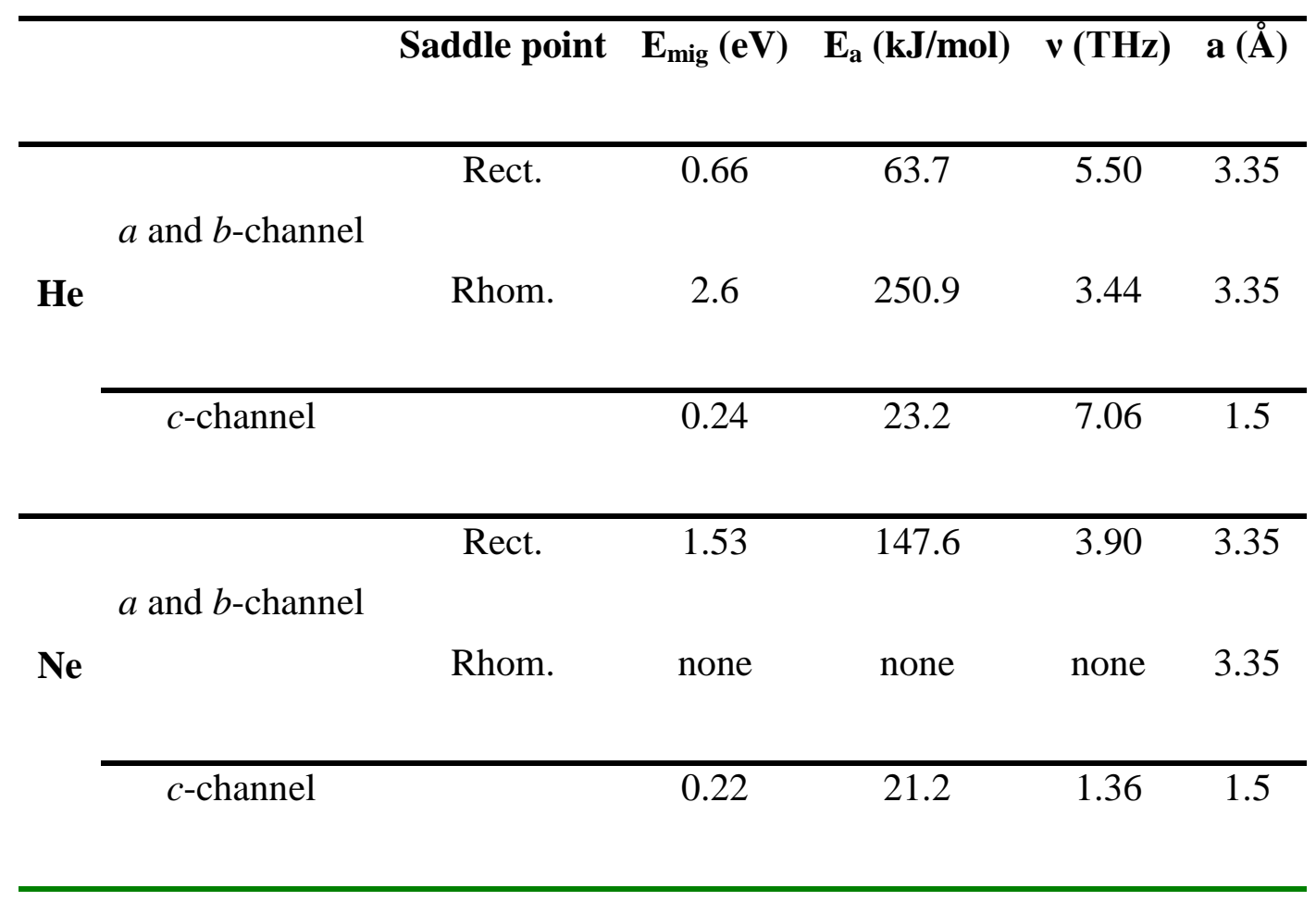


Table 4: Diffusion coefficients ( $D_{0}$ and Ea) along $a / b$ and $c$ axes for different percentages of obstruction, as shown in Figure 8.

\begin{tabular}{|c|c|c|c|c|c|}
\hline $\begin{array}{l}\% \\
\text { obstruction }\end{array}$ & $\begin{array}{l}\mathrm{D}_{0}(c) \\
\left(\mathrm{m}^{2} / \mathrm{s}\right)\end{array}$ & $\begin{array}{c}\mathrm{Ea}(c) \\
(\mathrm{kJ} / \mathrm{mol})\end{array}$ & $\begin{array}{c}\mathrm{D}_{0}(a / b) \\
\left(\mathrm{m}^{2} / \mathrm{s}\right)\end{array}$ & $\begin{array}{l}\mathrm{Ea}(a, b) \\
(\mathrm{kJ} / \mathrm{mol})\end{array}$ & $\begin{array}{c}\mathrm{Tc} \\
\left({ }^{\circ} \mathrm{C}\right)\end{array}$ \\
\hline 0 & $1.6 \times 10^{-7}$ & 23.1 & $1.5 \times 10^{-7}$ & 63.6 & -193 \\
\hline 1 & $4.6 \times 10^{-4}$ & 61.8 & $1.5 \times 10^{-7}$ & 63.6 & -100 \\
\hline 5 & $1.1 \times 10^{-5}$ & 60.6 & $1.5 \times 10^{-7}$ & 63.6 & -88 \\
\hline 10 & $2.3 \times 10^{-6}$ & 60.1 & $1.5 \times 10^{-7}$ & 63.6 & -83 \\
\hline 20 & $5.6 \times 10^{-7}$ & 61.9 & $1.1 \times 10^{-7}$ & 54.0 & -73 \\
\hline
\end{tabular}

Closure temperature calculated for a 100x100x200 $\mu \mathrm{m}$ prism, cooling rate of $10^{\circ} \mathrm{C} / \mathrm{Ma}$ and no ejection. 

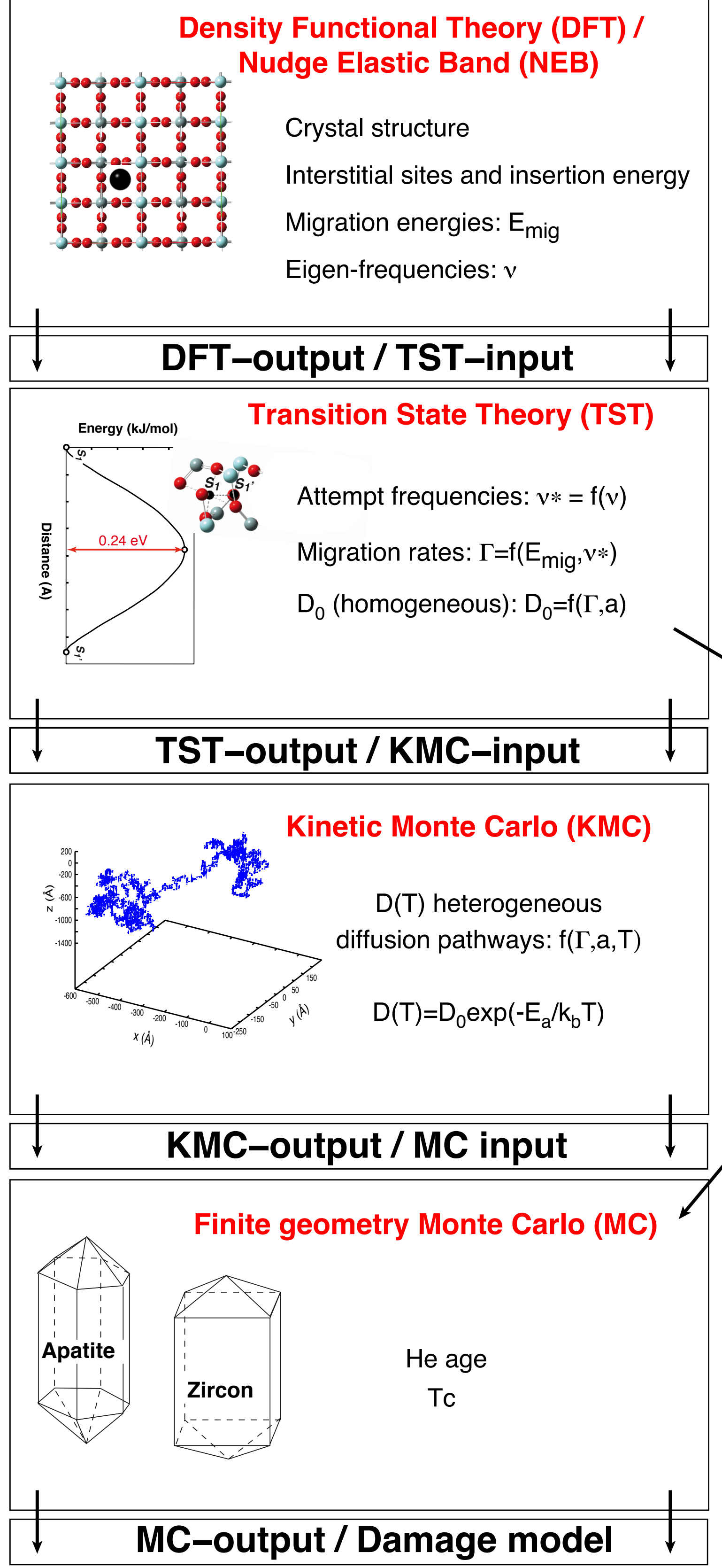
A

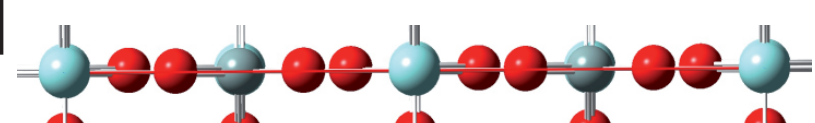
$88 \% 8$

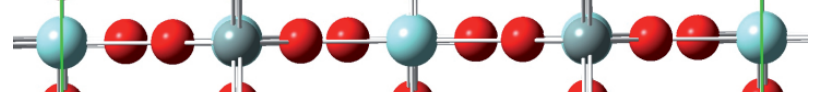
$8 \% 8 \%$ yougaeprages=

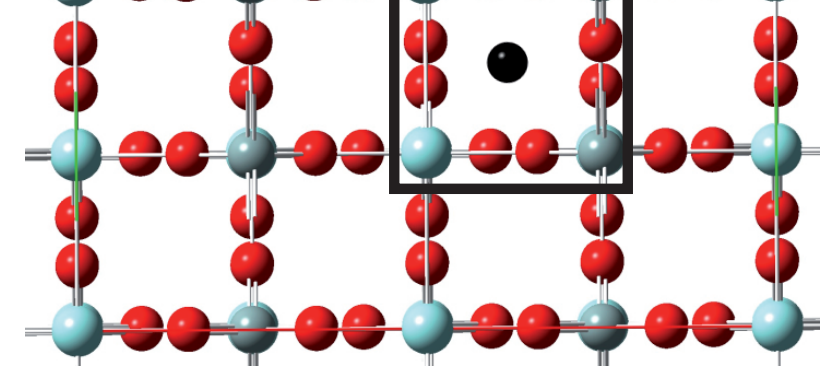

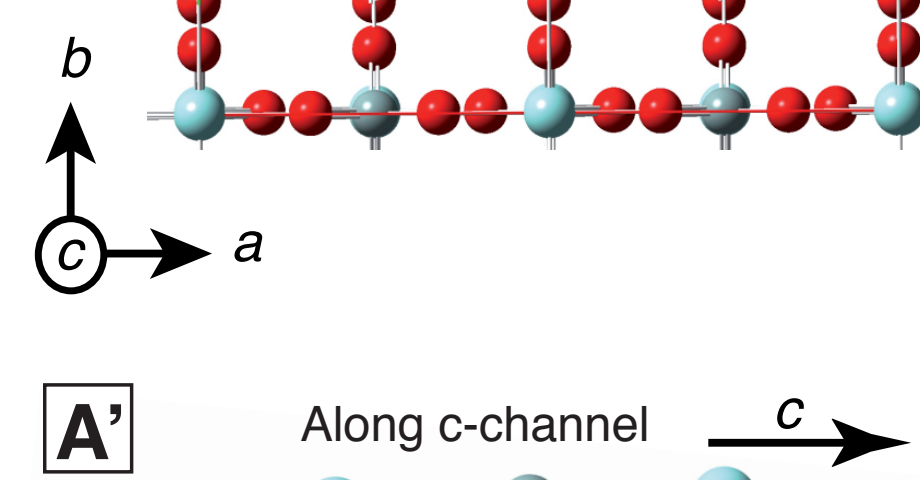

o zr

- Si

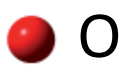
He $\mathrm{Ne}$

\section{B}

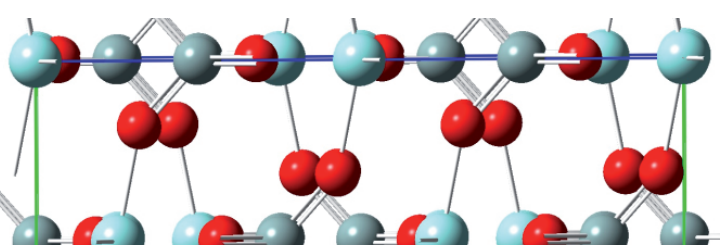
$\rightarrow C$ C

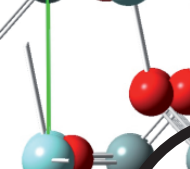
b 个 a) $\rightarrow c$ $\rightarrow C+2=$

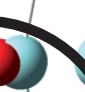

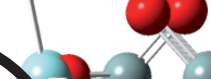

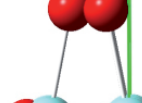

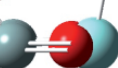
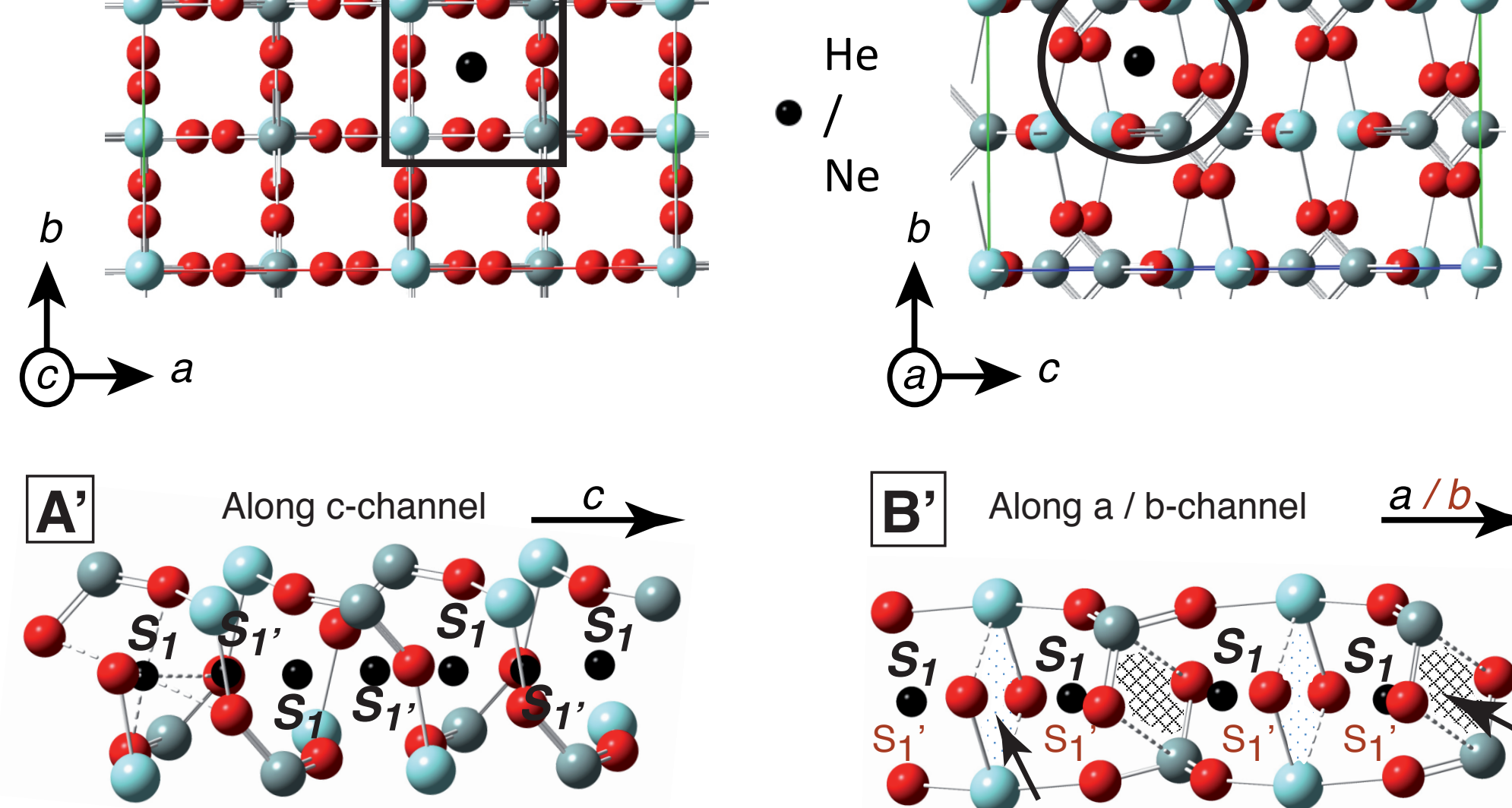

$\mathbf{B}^{\prime}$ Along a / b-channel $\stackrel{a / b}{\longrightarrow}$

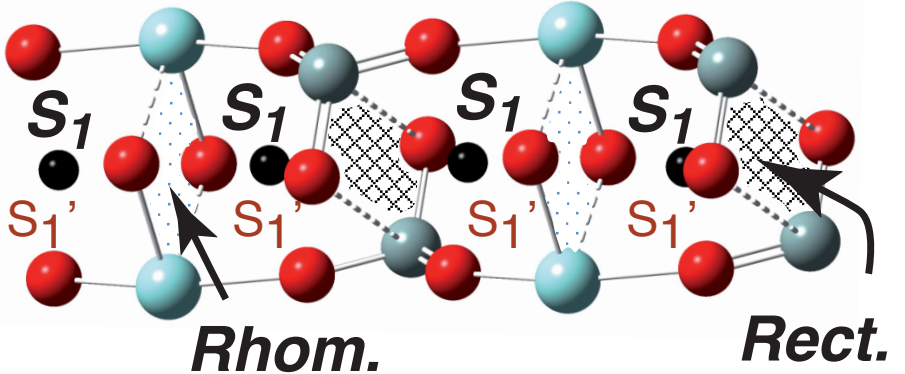




\section{Figure3}
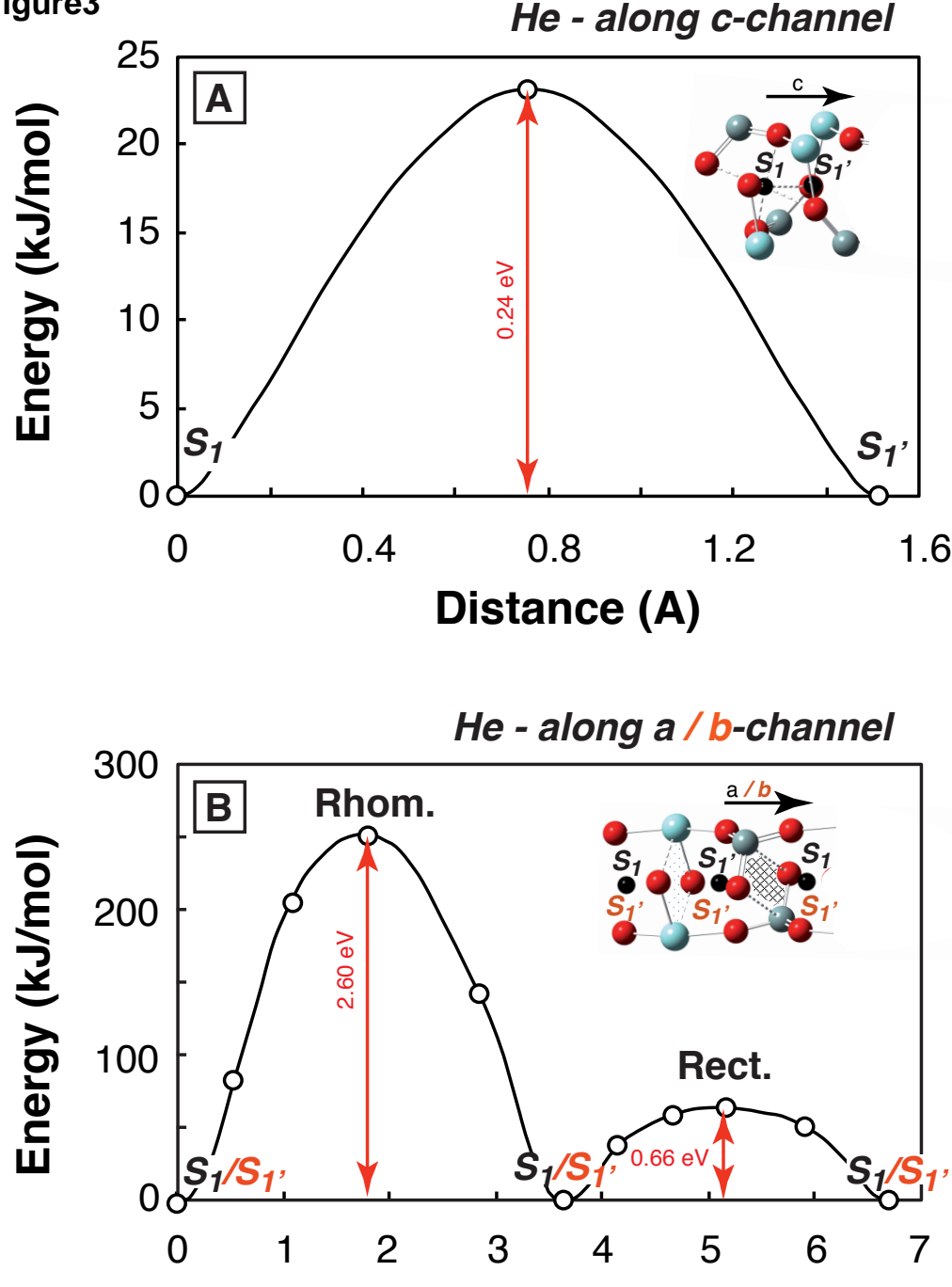

Distance (A)

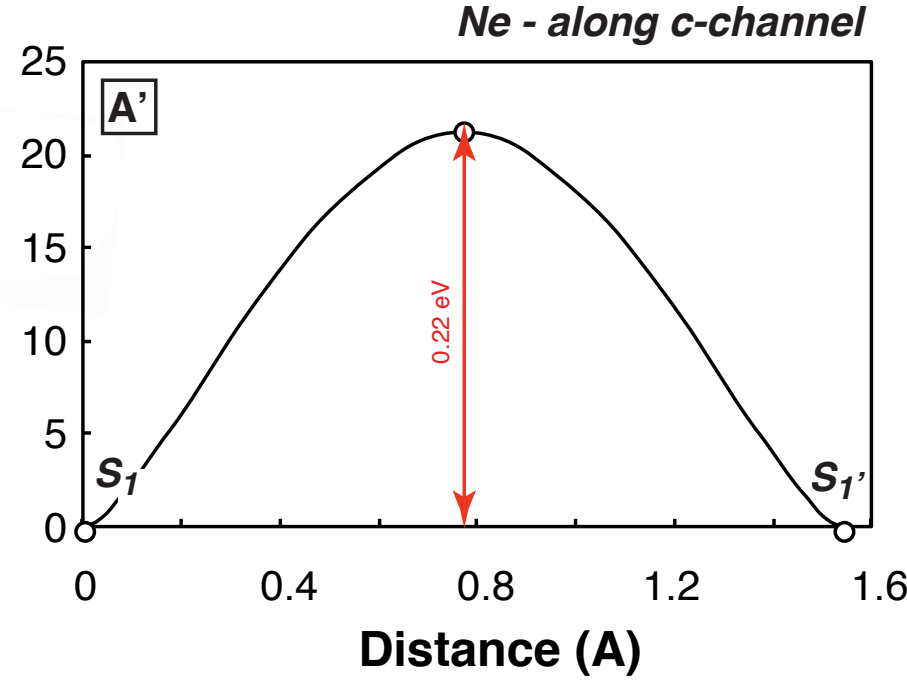

Ne - along a / b-channel

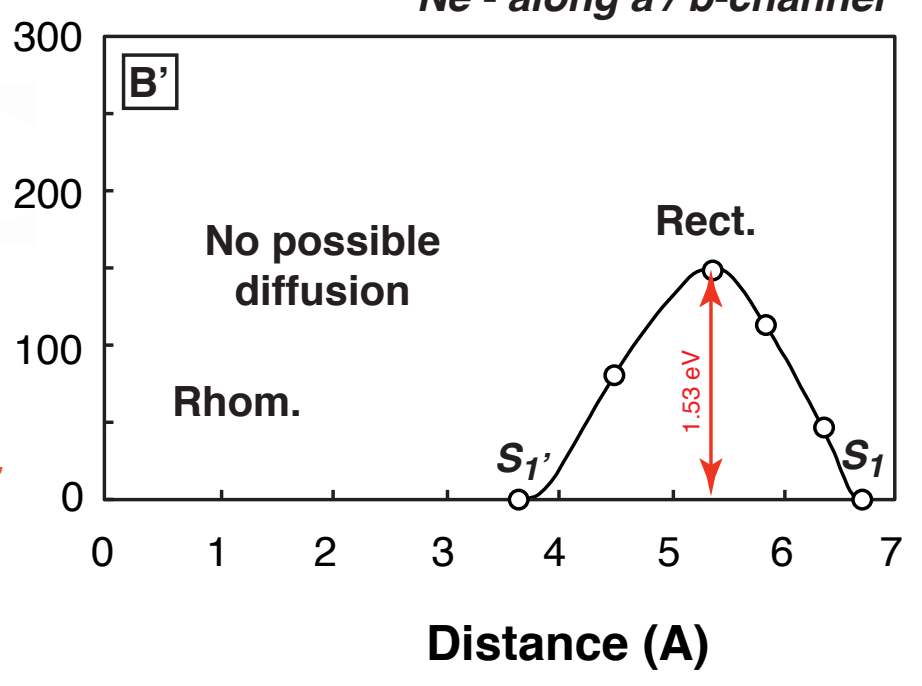



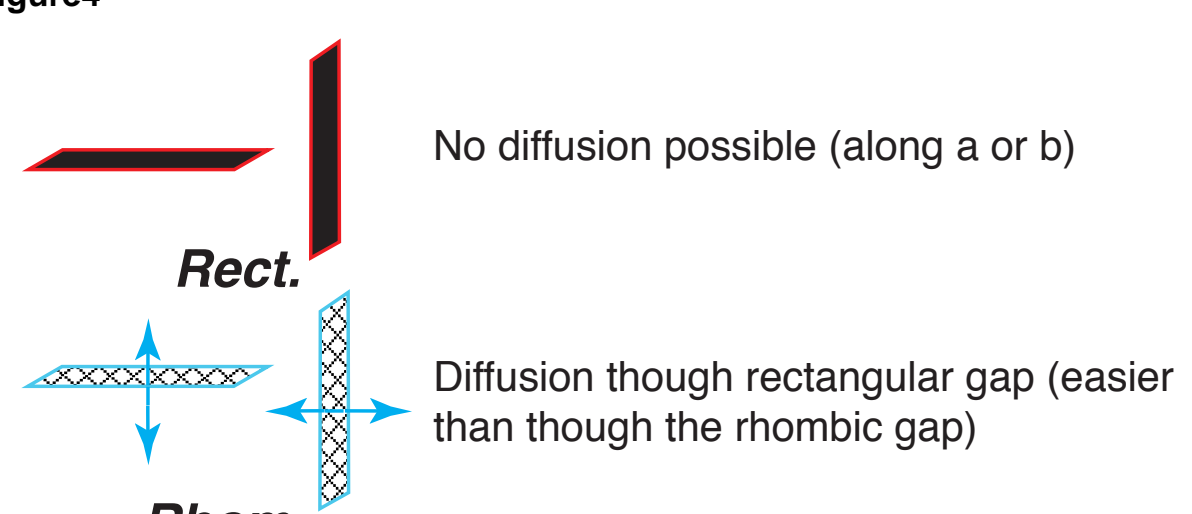

Diffusion though rectangular gap (easier

Rhom.

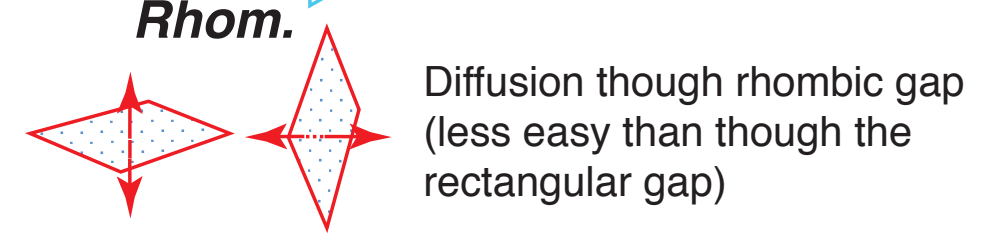

(less easy than though the rectangular gap)

\section{Rhom.}
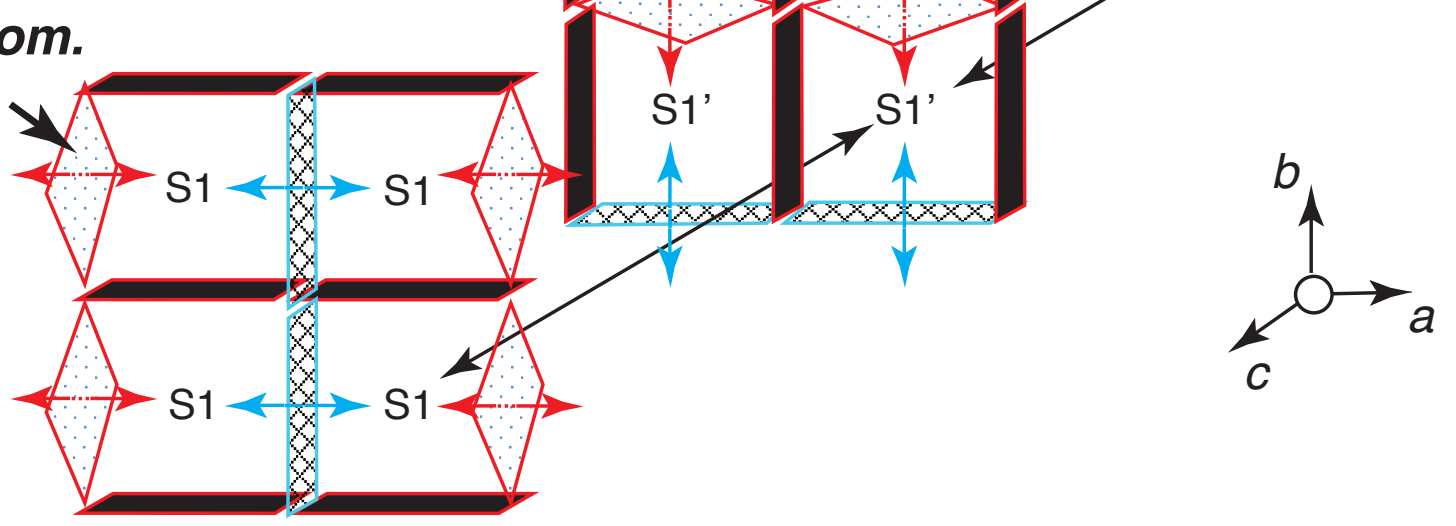

Along c-axis

diffusion between

S1 and S1' sites 

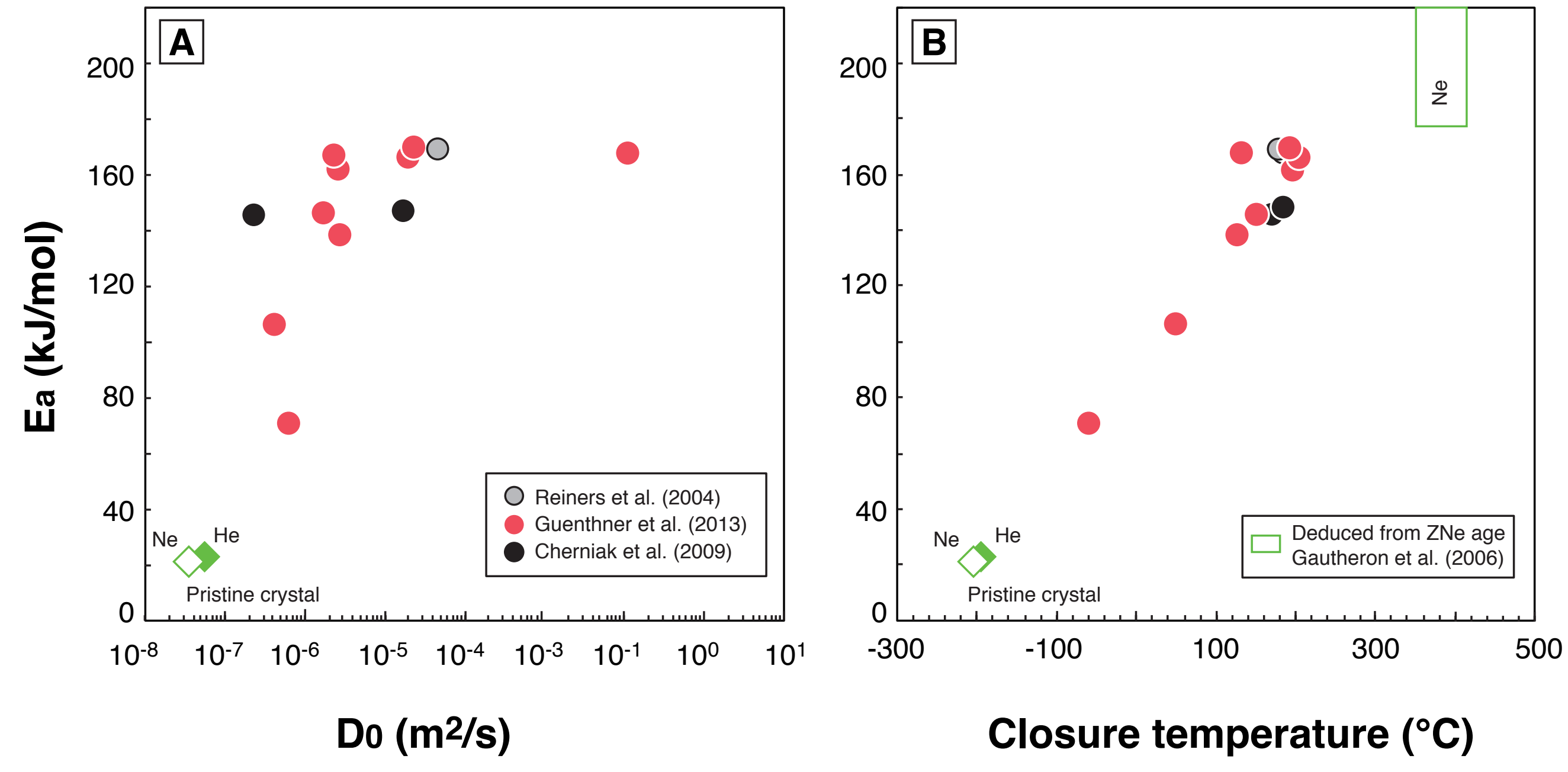


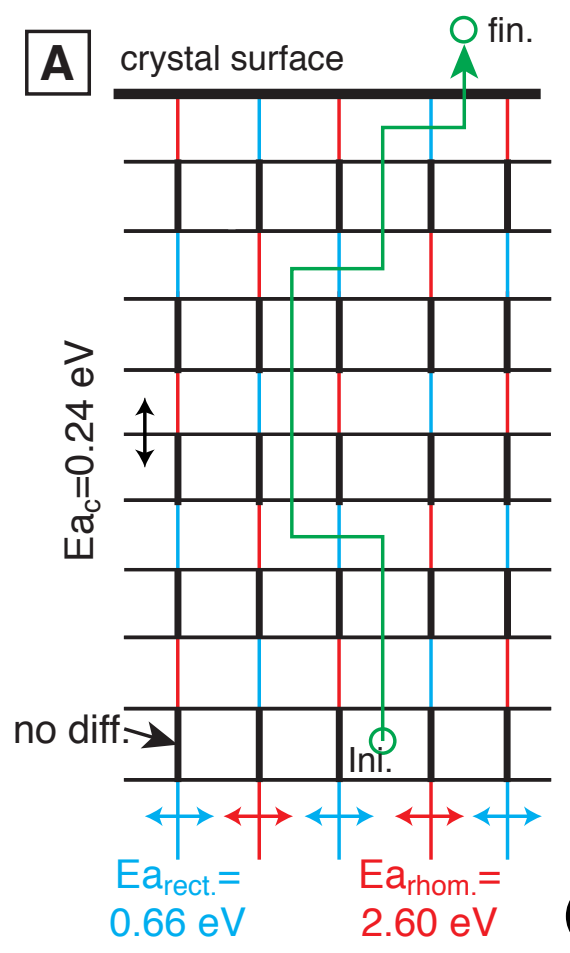

$B$

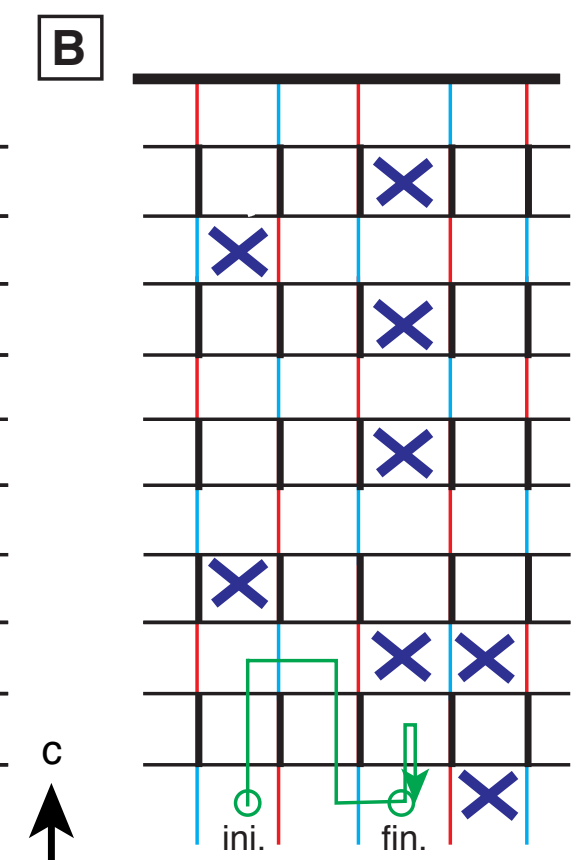

C

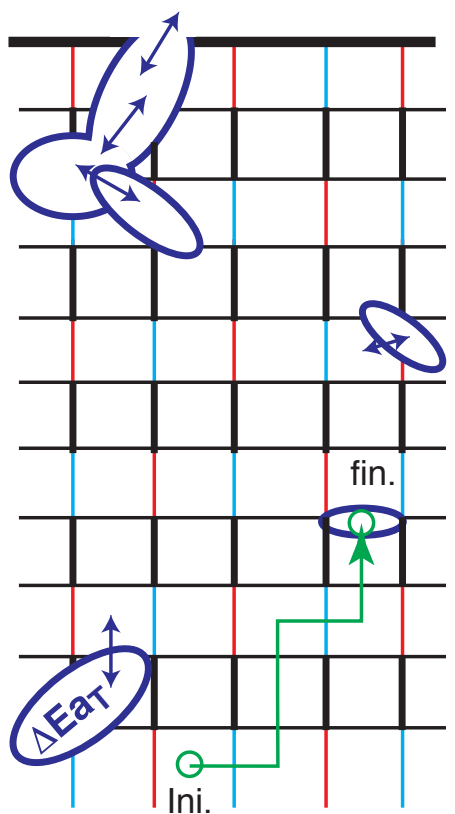
ini.

(b) $\rightarrow a$ 

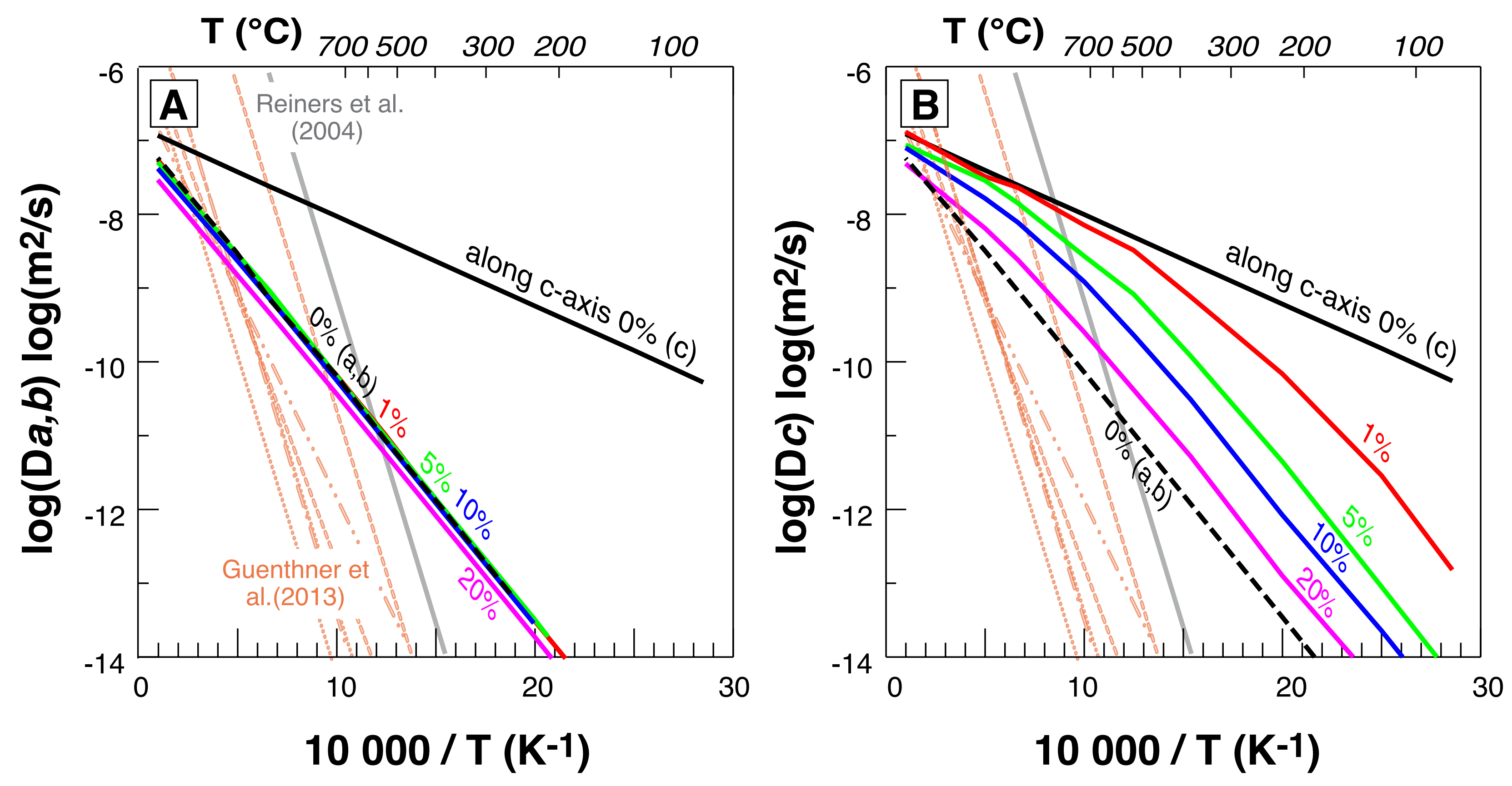


\section{Helium}
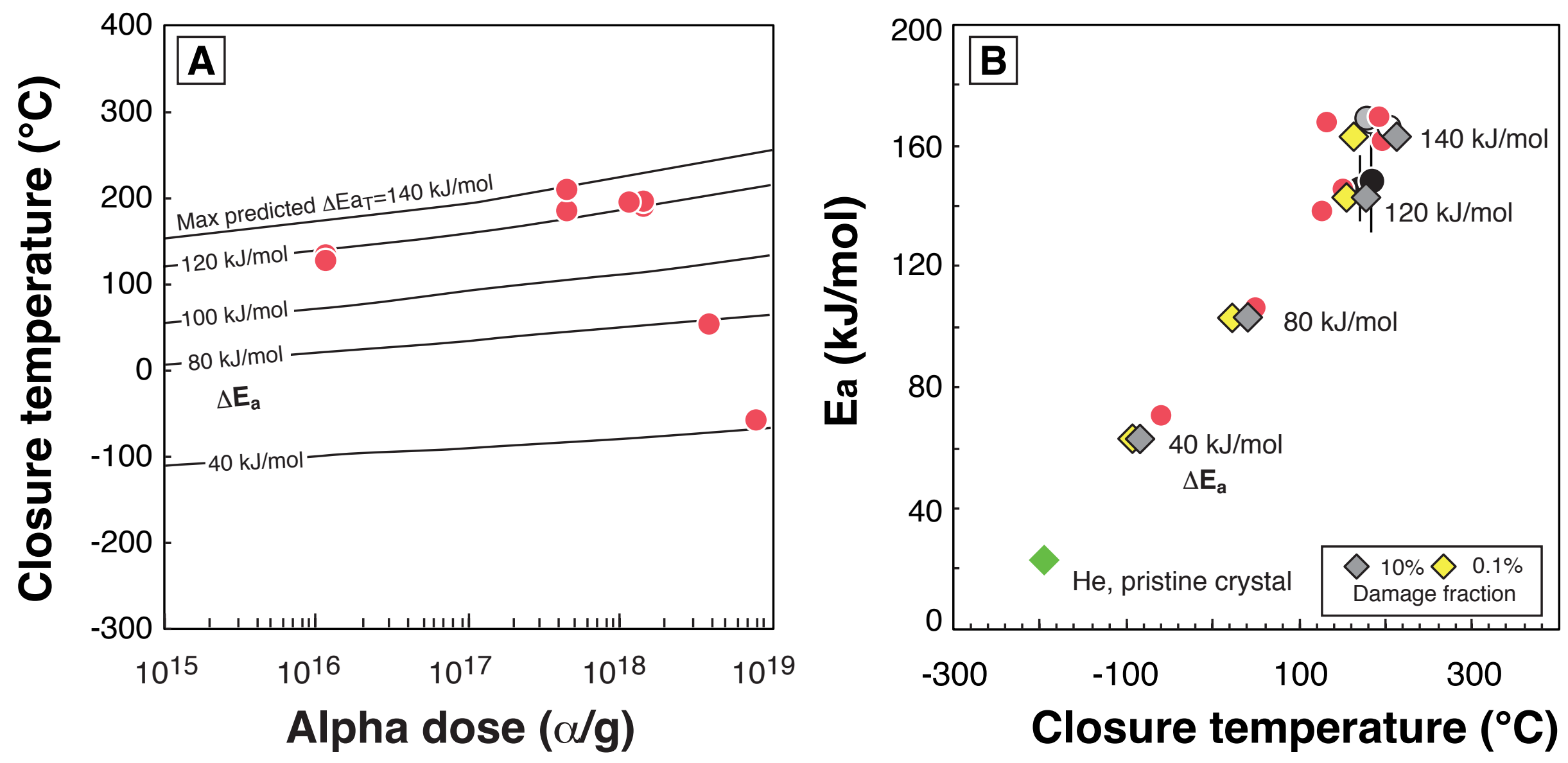

\section{Neon}
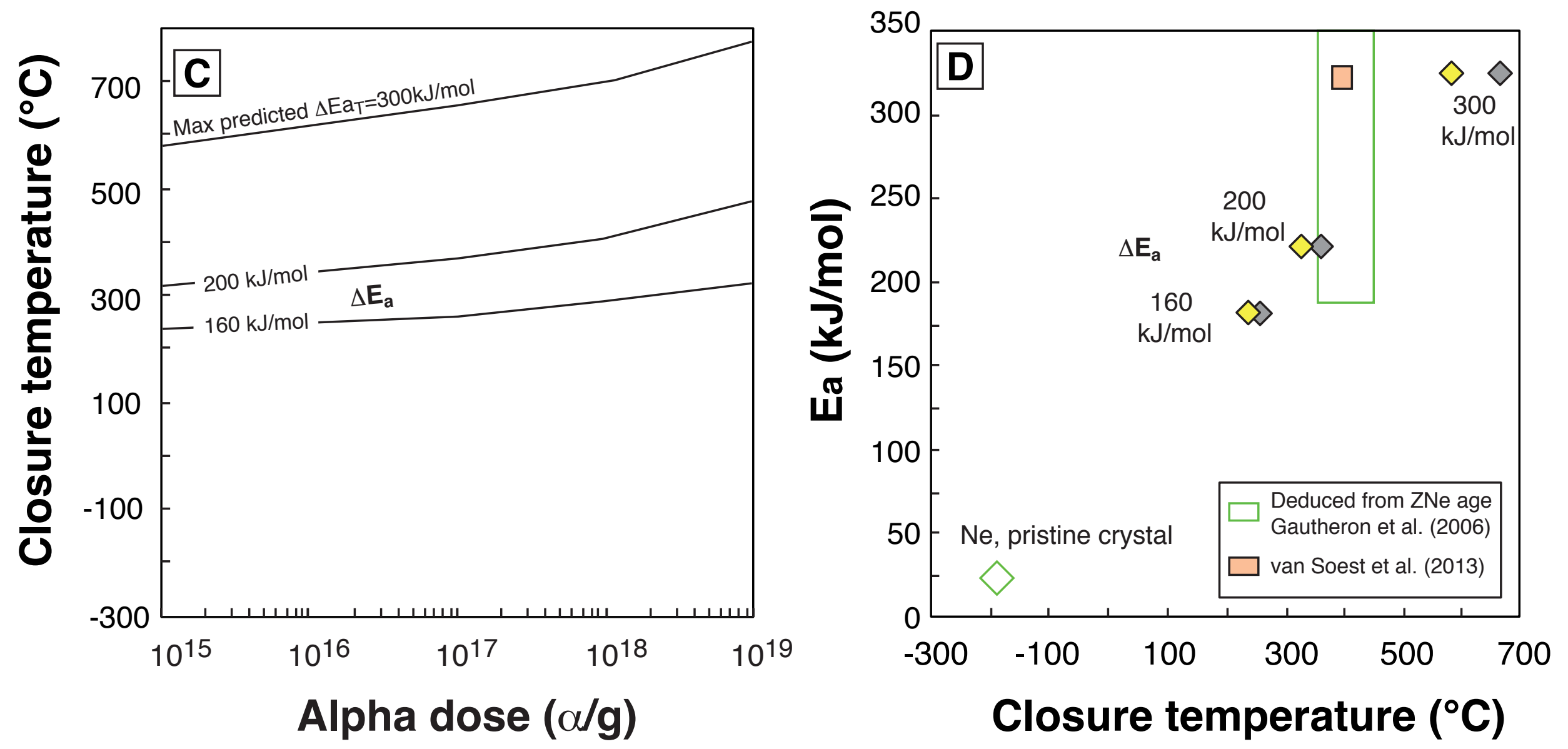


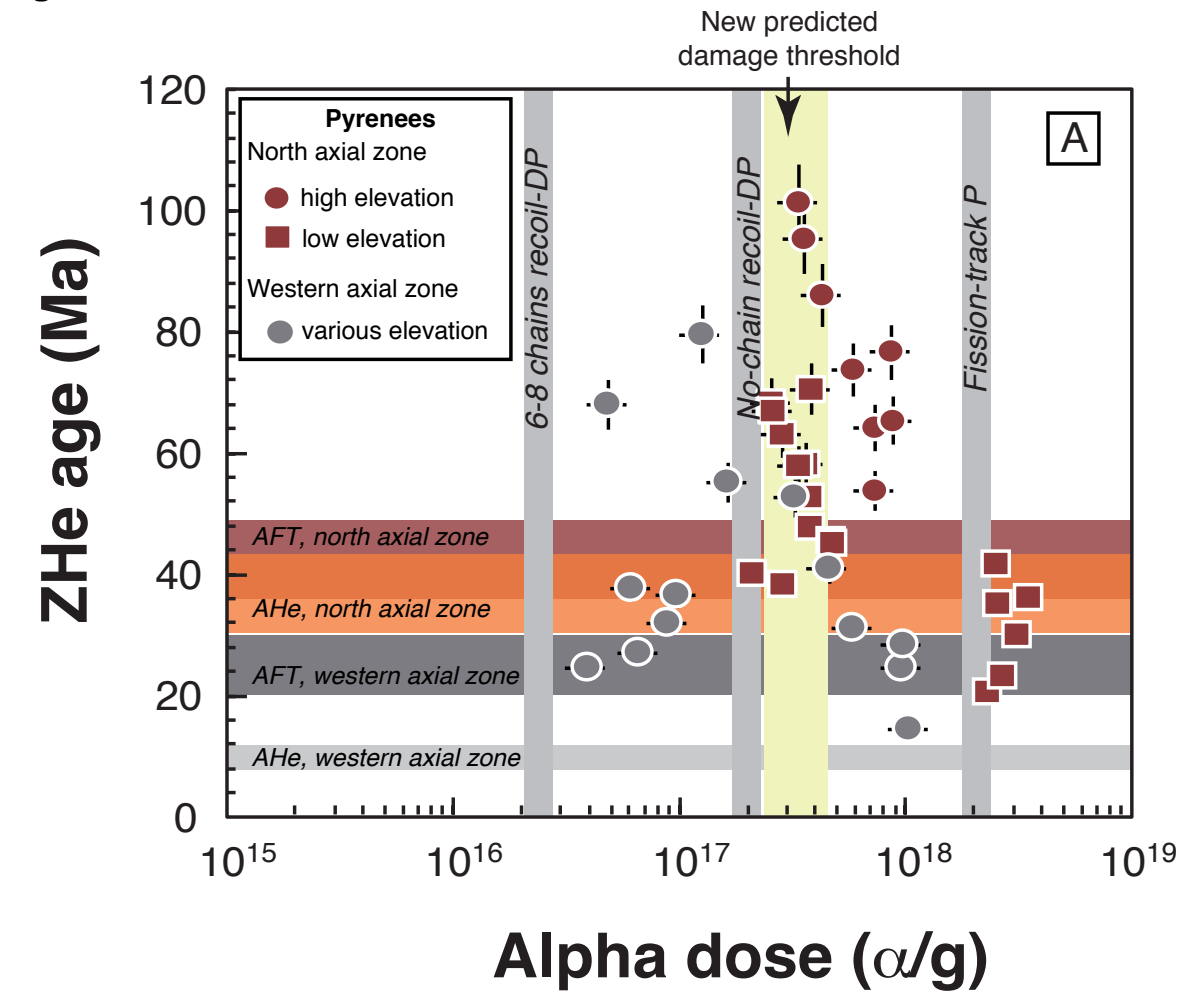

maximum possible current dose

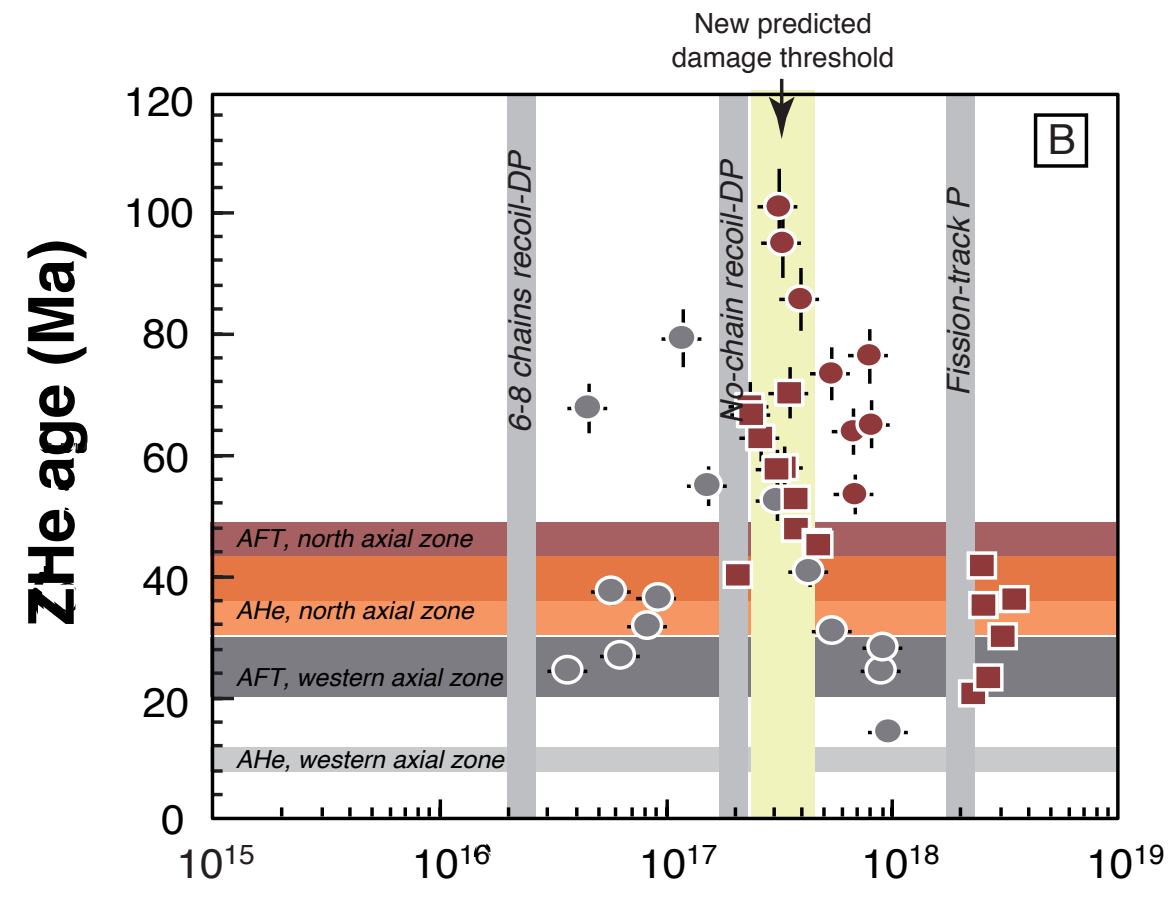

Alpha dose $(\alpha / g)$

maximum possible dose at estimated time of partial resetting 
A
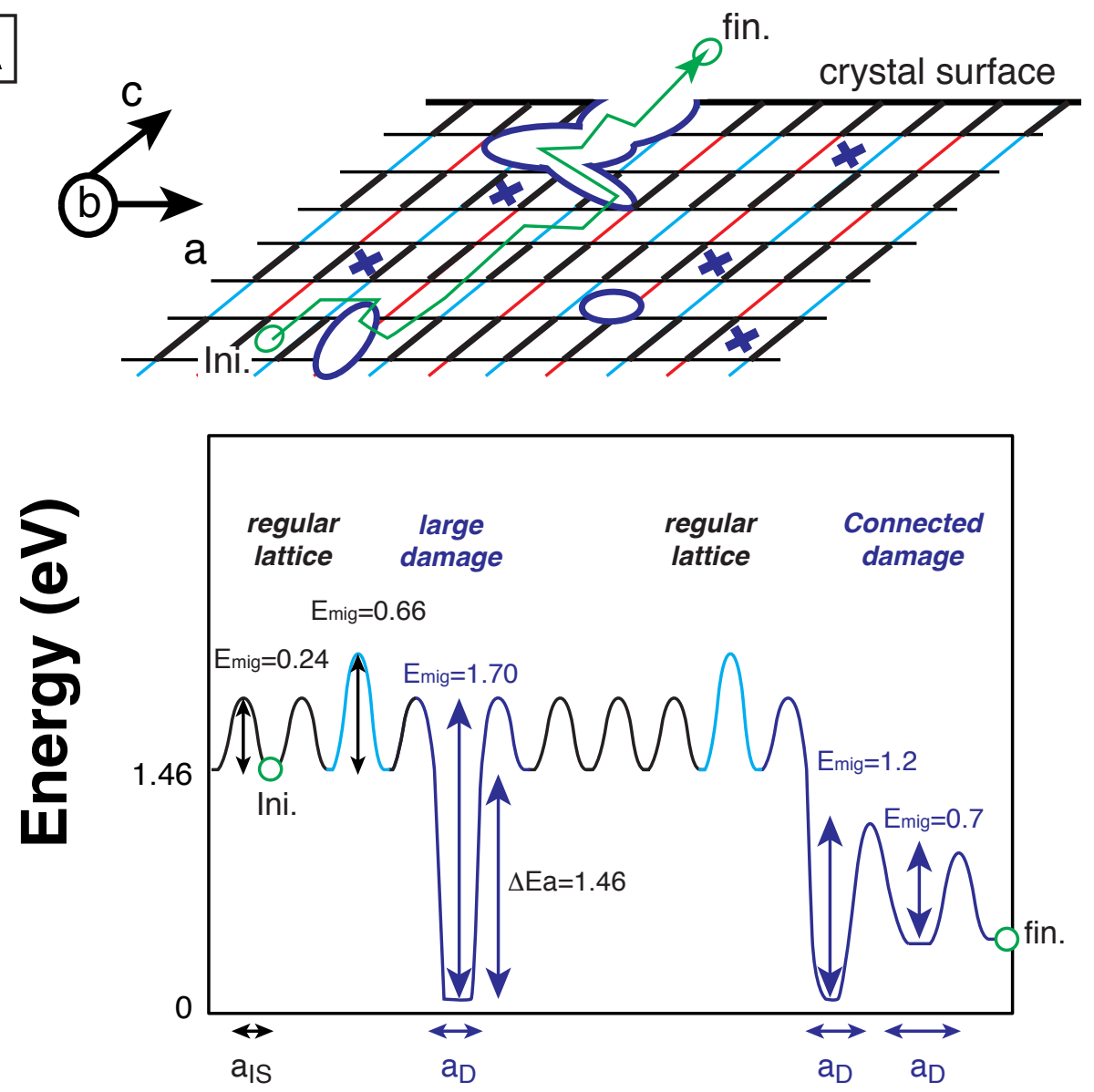

Diffusion path (n.u.)

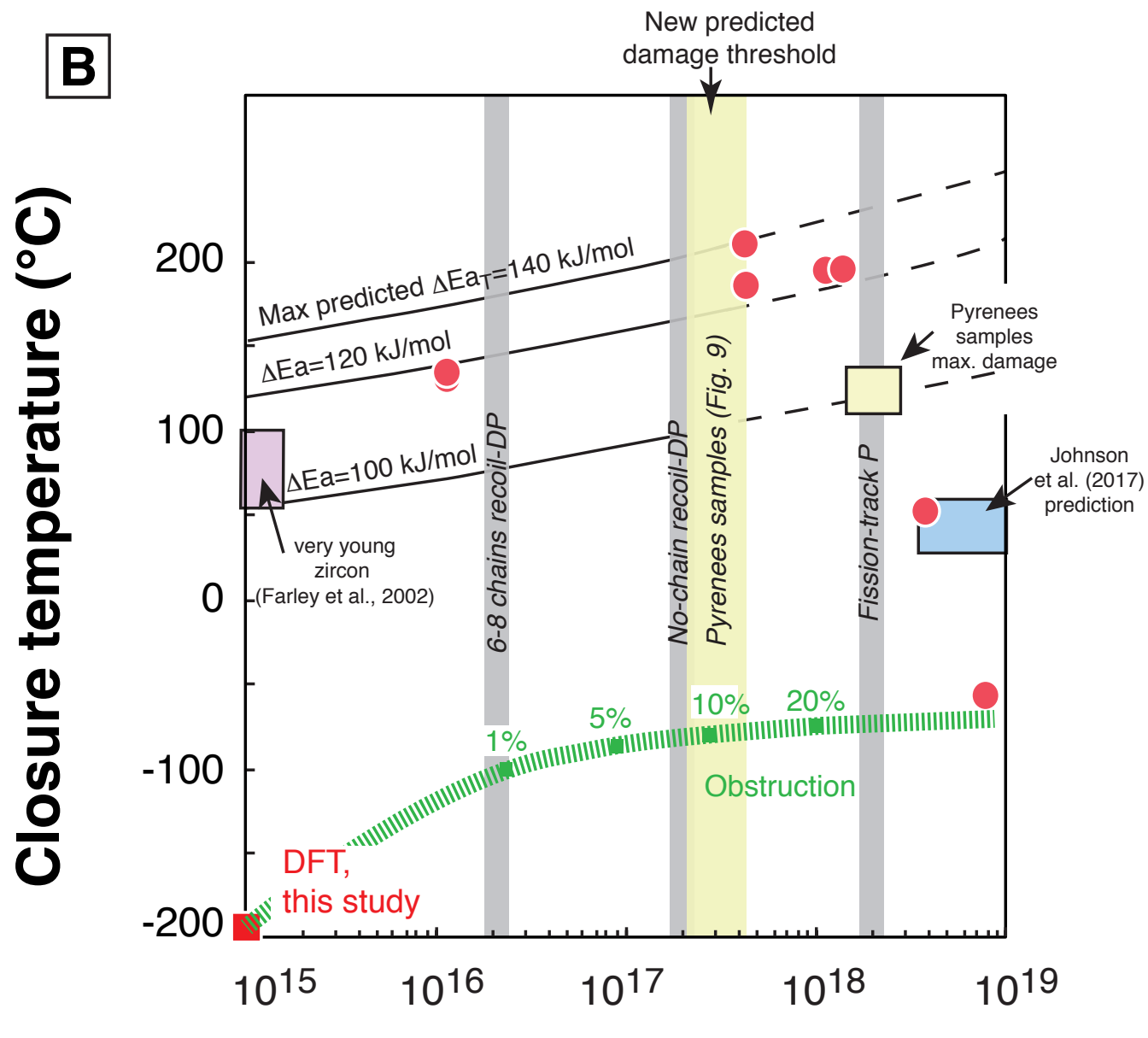

Alpha dose $(\alpha / g)$ 
